Bertille Lyonnet*, Xavier Faiure****

\title{
The Settlement Pattern of the Western Upper Khabur from the Old Babylonian Period to the End of the Mittani Era
}

The region surrounding the Khabur river in northern Mesopotamia has been the object of intense study for the last twenty years. Recent research has built upon the pioneering work on the most ancient periods by Baron von Oppenheim (I93I), Sir Max Mallowan (I936 and I937) and Van Liere and Lauffray (I954-I955), as well as the work on more recent eras by Father Poidebard (I934) and H. Dillemann (I962). A number of archaeological field surveys $^{\mathrm{I}}$ with widely different aims, scales, and methods have been carried out in the region, and over thirty tells, ranging from the pre-ceramic Neolithic to the Islamic period, including some of considerable size and importance, have been or are in the process of being excavated. Finally, the region has been a focus of research for the epigraphists of Mari because a major part of Zimrī-Lîm's archives (I8th century BC) deals with this area and offers abundant information about it.

The Upper Khabur clearly played an important role during the Mittani period: according to some scholars, Waššukanni and Taidu, Mittani's two capitals, were located in this region - one at the source of the Khabur at the site of Tall Fakhariya, ${ }^{2}$ and the other either along the Jaghjagh at Tall Hamidiye 3 or slightly more to the southeast at Tall Farfara.4 Nevertheless, we have few data regarding this period. The aim of this article is therefore to lay out the results of a field survey conducted under B. Lyonnet's direction in the western part of the Upper Khabur, 5 in the triangle between the present-day cities of Hasseke, Qamishly and Ras-el-Aïn, and to come to an understanding of the changes that occurred in the region between the Mittani period and the period that preceded it.

* Centre National de la recherche Scientifique (CNRS) Paris - UMR 7I92.

** Centre National de la recherche Scientifique (CNRS) Nanterre - UMR 704I.

I In particular, D. Oates I977, Röllig - Kühne I977-78 et I983, Fielden I981, Monchambert I984, Meijer I986, Stein - Wattenmaker I990, Lyonnet I992, Eidem - Warburton I996, Wilkinson 2000, Hole 2002-3, Ristvet Weiss 2005, Ur - Wilkinson 2008.

2 Opitz I927.

3 Haas - Wäfler I985.

4 Ristvet - Weiss 2005.

5 Lyonnet 2000 . 


\section{Brief summary of the methods used during the field survey and for the analysis of the data 6}

The field survey was purposefully carried out on a large scale in the hope of reconstructing the history of settlements in the region from the earliest origins to the present day. Given the considerable number of tells and the impossibility of visiting them all, we chose our sites from a map with a scale of $1: 200,000$, made during the French Mandate, spreading them across the region as evenly as possible so as not to leave any large blank zones, except in places where previous studies had already been carried out.7 The most prominent tells were systematically chosen, as well as smaller mounds located in unusual environments. In total, $6_{3}$ sites were visited. Their dimensions were measured by walking around the visible part of the tells. ${ }^{8}$ The potsherds were collected randomly but in large quantities (roughly fifteen tons); on the larger sites, it was carried out across the entire surface, which was successively divided into zones based on natural divisions visible to the naked eye. The satellite mounds surrounding the larger tells were for the most part also surveyed, except in cases where they were presently inhabited.

Unfortunately for our study of the Mittani period, the major settlement situated near Tall Beydar9 was too low to be seen on this map, and therefore was not surveyed. It is likewise unfortunate that Tall Fakhariya (T.6) yielded comparatively few potsherds (I3I.5 kg in ca. 3.5 ha): the site is largely occupied by cemeteries and quite 'urbanized,' 'о making it extremely difficult to survey. Furthermore, it was not our original intention to collect potsherds on it as it had already been excavated in the past, ${ }^{\text {II }}$ but rather to search for Khabur pottery at the request of the epigraphists working on the Mari texts. Our picture of that site is therefore necessarily incomplete.

We analyzed the ceramics that we collected in several phases. The first step of the study was dedicated individually to each site - and, when pertinent, to each zone. After sorting, counting, and describing all the potsherds, a first attempt at a general typology was made, and a certain number of redundant specimens were eliminated, although as a rule at least one sherd of each type was retained in the final selection. ${ }^{\mathrm{I2}}$ In a second step,

6 A more detailed discussion of this subject can be found in Lyonnet 2000 .

7 The site of Chagar Bazar, for instance, where Mallowan began his investigation of this region and which is now under excavation since our survey began, will not be found here.

8 While our measurements can be contested and are often smaller than those given by the excavators (see, for example, Tall Beydar = T. 15 in our survey), at least they are consistent, because they are all based on the same system.

9 Bretschneider 1997. Unfortunately, there have been no large-scale excavations of this site, and it is considered to be later (Iron Age) in Wilkinson 2000 and Ur - Wilkinson 2008.

IO See Lyonnet 2000a, 2I, 30 and 43.

II See McEwan et al. I958.

I2 Note, however, that the number of the potsherds of each type that was kept is not proportional to the number of sherds originally collected, but depended mainly on their state of preservation. This means that one has to return to the original notes kept for each site to get the true picture of how representative the retained potsherds are. 
a general typology was generated on the basis of the retained selected sherds. It covered all periods from the Ubaid $^{\mathrm{I} 3}$ up to the present day, and with it we made a first attempt at assigning periods - usually several centuries long - to each type or series of sherds. ${ }^{\mathrm{I}}$ In the third step, the retained materials from each period were given to graduate students, postdoctoral researchers, or specialists in the relevant fields to refine the dating of each series and to study the distribution of settlements in preparation for the final publication. Finally, a comprehensive list of the provenance of the retained potsherds from each series, along with a precise description of each type and series including photos and drawings, was compiled.

For various reasons, certain participants have been unable to finish the work they began and, as a consequence, the results of this field survey have not yet been fully prepared for publication ${ }^{15}$ - although we still intend to publish them as soon as possible. However, the data from the first half of the second millennium BC, given to Xavier Faivre, have already been studied in greater details in his doctoral thesis (also unpublished). His results are given in this article and we have added as a complement to his work the quantitative data coming from the first step study of the field survey.

Before we go into the full details, however, it is important to summarize a certain number of facts concerning the environment in the region and how it relates to the major discoveries made during this field survey about the general settlement pattern.

\section{Environmental conditions in the region}

The region of the Upper Khabur, surrounded on three sides by mountains of modest size (Tur Abdin to the north, Jebel Abd-el Aziz to the southwest, and Jebel Sinjar to the southeast), appears in the form of a wide undulating plateau crossed by wadis with irregular flow. The majority of these wadis flow from the Tur Abdin in the north either directly into the Khabur or indirectly by way of the Jaghjagh. Due to a series of karstic springs in the Ras-elAin region, the Khabur is the only waterway in the region today that flows year-round. The relatively uniform character of the landscape in this area has led many archeologists to consider it as having always been a single geographic entity.

Except for several zones shaped by basalt flows, the whole of the region is, at least in appearance, favorable for agriculture without irrigation. This, of course, depends essentially on the annual amounts of rainfall. Indeed, a 30omm isohyet, below which non-irrigated agriculture is usually risky, currently crosses the region from the northwest to the southeast, running parallel - a few kilometers to the north - to the upper course of the

I3 The older periods of the Neolithic (Proto-Hassuna and Halaf) were treated separately, see Lyonnet 2000 .

I4 These were the results that were shown in table 4 of Lyonnet 2000 .

I5 Only volume I has appeared thus far (Lyonnet 2000b), three more volumes are in preparation. 
Khabur. This line is however unstable and fluctuates more or less toward the northeast or southwest, depending on the year. As a result, the Khabur, though the only perennial body of water in the region, is located in a zone that is generally too dry for major agricultural production. In fact, the sites situated along its course between Ras-el-Ain and Hasseke are for the most part of a very modest size compared to those that are found along the Jaghjagh.

Environmental studies carried out during the field survey ${ }^{\mathrm{I} 6}$ demonstrated certain climatic variations over time: a gradual desertification began in the fourth millennium BC and reached its apogee at the end of the third. This was followed by an arid and windy period which lasted until the middle of the second millennium BC, at which point the region seems to have returned to a more stable climate, close to present conditions.

\section{Major results of the field survey concerning the early periods}

One of the main results of the survey has been the identification of significant differences in the distribution of population within the study area throughout its history. These results have been considered as related to the above-mentioned division between the relatively humid zone in the northeast and the comparatively drier zone in the southwest of the Upper Khabur triangle.

Since the Pre-Halaf period, the southwestern part of the region has always had a lesser density of settlements than the northeast, as well as settlements of smaller size (Nieuwenhuyse 2000, see maps at figs. 73, 74, 76, 77). ${ }^{77}$ Until the end of the fourth millennium, it does not appear that this differentiation was reflected in the ceramic materials. But this begins to change during the third millennium: all the sites in the northeast yielded abundant quantities of potsherds painted or incised with "Ninivite 5" type decorations, while those to the southwest yielded almost none. Similarly, at the beginning of the second millennium, the pots painted in the "Habur pottery" style appear in abundance in the northeast, while only a few examples can be found in the southwest. This division in pottery styles between the two regions has been confirmed recently by other surveys ${ }^{18}$ as well as by excavations at a certain number of sites: in the southwest, the percentages of Ninivite 5 pottery unearthed are insignificant, while no "Habur" level at all has been discovered (see for instance Tall Beydar), in marked contrast to sites in the northeast (like at Tall Arbid, for example).

In so distinctly separating the northeastern part from the southwestern of the western Upper Khabur, we might add that the latter is more closely related to the so-called Middle

I6 Courty I994.

I7 On this topic also see Nieuwenhuyse - Wilkinson 2008.

I8 Wilkinson 2000; see Ur - Wilkinson 2008 for the region around Tall Beydar. 
Khabur region, where numerous excavations and surveys have revealed similar pottery styles and the same specific 'absences' of "Ninivite 5" and "Habur" pottery. ${ }^{19}$

As far as the first half of the third millennium is concerned, this particular distribution of pottery together with, in the southwestern area, on the one hand, the outlying position of the Kranzhügel of Tall Beydar in comparison to the other settlements of this type, and with, on the other hand, the discovery of many small settlements dedicated to the storage of grain but with only few living spaces has led us to advance the hypothesis that there was a close relationship between this region and the more arid steppe between the Balikh, the Euphrates, and the Khabur and to consider that it was the domain of pastoralists who raised flocks, cultivated fodder, and lived a partly nomadic life between the river and the steppe. ${ }^{2 \circ}$

As far as the first half of the second millennium is concerned, it has been proposed that even more nomadic peoples inhabited this region, leaving almost no trace of their existence apart from references in the texts from Mari..$^{2 \mathrm{I}}$ This hypothesis has received strong support from J.-M. Durand, who has come to consider the southwestern region as corresponding to the 'steppe of the wadis' of the Ida-Maras, where Bensimalite groups came to graze their flocks. ${ }^{22}$

However, the distinction between the northeast and southwest is not always as clear across history, perhaps because of a general southward movement of the $300 \mathrm{~mm}$ isohyet, or perhaps for other, more political reasons. Our general analysis of the ceramics was suggesting for instance that the region to the southwest was resettled from the Mittani period onwards. ${ }^{23}$ But, given that the pottery from this period was found in much smaller quantities than the pottery usually attributed to the Old Babylonian period, we were awaiting the final results of our in-depth study to confirm this claim.

\section{A more detailed study of settlement during the first half of the second millennium}

It is not always easy to determine exact dates for pottery collected during a field survey: in fact, it is rare for scholars to attempt to make chronological distinctions of less than 400 or 500 years based on such a data set. ${ }^{24}$ There are many ceramic types that continue across (or straddle) two politically different time periods, and the military and political conquests

I9 See, for example, Kulemann-Ossen 2009 regarding the first half of the second millennium based on the survey of the Middle Khabur. Tall Taban is the only site that could have been occupied during the second millennium until a phase pre-dating the Middle Assyrian period (Shibata 2009).

20 Lyonnet I998; 200I; 2004; 2009.

2I Lyonnet 1996.

22 Durand 1998; 2004

23 Lyonnet 2000a, I9 and tableau 4, p. 33-37.

24 See, for example, Wilkinson 2000 and Ur - Wilkinson 2008, who do not distinguish the Mittani period from the Khabur period. 
that are conventionally used to mark history are rarely followed by an immediate or complete change in pottery styles.

Although the pottery of the Old Babylonian period (Khabur pottery) is very distinctive and can be clearly distinguished from that of the third millennium, certain characteristics, like a decorative design of horizontal bands and triangles, are still present in the Mittani period and in some cases even later. The shape of the pottery as well as the clay from which it was made, usually allow us to distinguish different periods, but certain types which started during the Old Babylonian epoch, like the flattened, horizontal rim, last until the beginning of the Mittanian era. Similarly, some types that appear during the Mittanian period survive well into the Middle Assyrian one. Added to this problem of the long duration of pottery types is the fact that some decorative designs, like the painted triangles, may also date to the end of the fourth or the beginning of the third millennium, ${ }^{25}$ and that a certain number of Mittani pottery vessels take up forms known from a still older period (fourth millennium). ${ }^{26}$

All of these problems could be easily resolved if each site were only inhabited during one period, but this occurs extremely rarely in this region. It is therefore necessary to regularly check the original notes taken for each site and for each zone in order to decide the relative importance of each occupation period in case of doubts, or at least to underline the problem when it is impossible to decide.

In the preliminary study of the material initially collected during the survey, a dividing line was proposed between the Old Babylonian period and Mittani period; the most densely inhabited sites for each period (as compared to less populous or uninhabited sites from the same era) were identified but not numerically ranked. ${ }^{27}$ This work was later refined by $\mathrm{X}$. Faivre, ${ }^{28}$ who carried out his chronological identifications using only the potsherds that were actually retained in the third step of analysis. This allowed him to propose a more precise periodization, in which he separated the Old Babylonian phase from a "late Khabur/ early Mittani" phase, which was in turn followed by the Mittani phase. The results he reached are given below $(\mathbb{A}$ A). Further adjustments have been made using quantitative analysis based on the data from the first step of the study, that is on the totality of the collected material ( $\mathbb{B}$ B).

The results brought to light by these two approaches generally accord with each other as well as with the data supplied by a study on the population density at each of the surveyed sites. Nevertheless, it seems important to give individual accounts of these methods so as to show how they inform each other and how they will allow us in the end to discern more precisely the changes in settlement between the two major periods with which we are concerned.

25 See, for example, Koliński 2007.

26 Oates 1987.

27 Lyonnet 2000a, table 4, p. 33-37.

28 Faivre I999. 


\section{A Study of the retained materials}

\section{A.1 The Old Babylonian Period (Table I and Fig. 1)}

Faivre identified the typical assortment of pottery for this period on the basis of comparisons made between different sites, in particular with phase IX of Tall Muhammad Diyab. From the materials retained after the initial study, Faivre determined close to 4,000 sherds, ${ }^{29}$ separated into 156 different pottery series by shape and decoration, ${ }^{30}$ to be characteristic of this period. Table I gives a list of the sites at which these sherds were collected, in decreasing order of quantity of sherds retained (left column).

In his study, nearly all the sites (6o of the 63 excavated) appear to have been inhabited during this period. But we see right away that more than half of these 4,000 potsherds $(2242+52$ ?) were gathered from a handful of sites, three of which are located in the northern part of the Wadi Aaoueij basin (T. 3I, 33, 32 in order of the size of the yield), two in the basin of a wadi without name between the Wadi Dara and the Jaghjagh (T. 45 and 42), and one on the Wadi Dara (T. 38). ${ }^{\text {I }}$ Four further sites indicate relatively large settlements (more than Ioo sherds retained at each) - T. 24, 25, 34, 43 - while another eleven seem to have been only lightly inhabited, indicated by the retention at each of between 45 and 90 sherds for this period (T. 7, I3, I8, 28, 40, 4I, 44, 53, 54, 56, 70). The remaining sites yielded only a very modest quantity of potsherds (fewer than 30). It is to be noticed that the I4 sites situated along the Khabur (I to II, 49, 50, 6I) are all part of this last category except for T.7, situated at the source of the Khabur near Fakhariya.

Faivre's study did not calculate the population density, as based on settlement size, at each of these sites. If we continue to base our projections solely on the number of potsherds retained during the third step of our study, this changes the classification that was previously established (right column). Among the ten most densely occupied sites in this period, seven cover an area of less than I.5 ha: they are, in order of classification, T. 43, 32, 23, 25, 34, 40, 39. Another two have an area of between I.5 ha and 4 ha (T. 3I and T.38). And only one, T. 33, covers a surface area of more than 4 ha. The overall picture of population distribution is mostly unchanged by this calculation, as eight of these ten sites are located in the northern part of the surveyed zone and none is on the Khabur (Fig. I). We note, however, that the most densely occupied site, T. 43, had before been ranked as the tenth largest, and that T. 45 and 42 move from third and fourth largest to thirteenth and nineteenth respectively, while T.23 goes from twenty-third to third.

29 Faivre I999, where the numbers vary: vol. I, p. 42 (4044 = total of the numbers indicated in the table p. 39-42) and p. IIO $(3959+\mathrm{I} 29=4088)$ and vol. II, fig. I2O (= 3977 + I29?).

30 Series are more precise than types, and this explains that they are more numerous.

3I Faivre I999, fig. I20. 
Table I | List of sites that yielded potsherds dating to the Old Babylonian period, taken from the sample retained after the first selection. They are ordered according to the quantity of potsherds retained (left column) and by density of population (right column). Potsherds followed by a question mark were not counted.

\begin{tabular}{|c|c|c|c|c|c|c|}
\hline \multicolumn{2}{|c|}{$\begin{array}{l}\text { Ranking of sites according } \\
\text { to number of retained pot- } \\
\text { sherds }\end{array}$} & \multirow[t]{2}{*}{ Drainage Basin } & \multirow[t]{2}{*}{ Surface } & \multirow{2}{*}{$\begin{array}{l}\text { Number of } \\
\text { retained pot- } \\
\text { sherds from } \\
\text { the Old } \\
\text { Babylonian } \\
\text { Period } \\
\\
\text { Total = } 3977 \\
+ \text { I29? }\end{array}$} & \multicolumn{2}{|c|}{$\begin{array}{l}\text { Ranking according to } \\
\text { population density }\end{array}$} \\
\hline Ranking & Site & & & & $\begin{array}{l}\text { No. of pot- } \\
\text { sherds/ha }\end{array}$ & Ranking \\
\hline I & 3I (Tell Dibak) & $\begin{array}{l}\text { Wadi Aoueij, left } \\
\text { bank }\end{array}$ & ca. 2,30 ha & $54 \mathrm{I}+4 ?$ & $235,2 \mathrm{I}$ & 4 \\
\hline 2 & 33 (Tell Hanoua) & $\begin{array}{l}\text { Wadi Aoueij, left } \\
\text { bank }\end{array}$ & ca. 3,9o ha & $404+5 ?$ & IO3,58 & IO \\
\hline 3 & 45 (Tell Ahmar) & $\begin{array}{l}\text { Unnamed Wadi / } \\
\text { Jaghjagh }\end{array}$ & $4,9 \circ \mathrm{ha}$ & $397+\mathrm{I} 3 ?$ & $8 \mathrm{I}, 02$ & I3 \\
\hline 4 & 42 (Tell Arbid) & $\begin{array}{l}\text { Unnamed Wadi / } \\
\text { Jaghjagh }\end{array}$ & 7 ha & 356 + I0? & 50,85 & 19 \\
\hline 5 & 32 (Tell Kdich) & $\begin{array}{l}\text { Wadi Aoueij, left } \\
\text { bank }\end{array}$ & ०,8० ha & $285+9 ?$ & 356,25 & 2 \\
\hline 6 & $\begin{array}{l}38 \text { (Tell Aïn el } \\
\text { Qurd) }\end{array}$ & Wadi Dara & 2,42 ha & 259 + II? & 107,02 & 9 \\
\hline 7 & 25 (Tell Arade) & Wadi Zerkan & I ha & $\mathrm{I} 42+5 ?$ & $\mathrm{I} 42$ & 5 \\
\hline 8 & 34 (Tell Atach) & $\begin{array}{l}\text { Unnamed Wadi / } \\
\text { Jaghjagh }\end{array}$ & $\mathrm{I}, 20 \mathrm{ha}$ & $\mathrm{I} 4 \mathrm{I}+9 ?$ & II7, 5 & 6 \\
\hline 9 & 24 (Tell Hermel) & Wadi Zerkan & 2 ha & $\mathrm{I} 35+\mathrm{I} ?$ & 67,5 & 15 \\
\hline Io & 43 (Tell Farho) & Wadi Dara & ca. $0,3 \circ$ ha & IIO & 366,66 & $\mathbf{I}$ \\
\hline II & 4I (Tell Baqar) & $\begin{array}{l}\text { Unnamed } \\
\text { Wadi / Dara }\end{array}$ & $2,20 \mathrm{ha}$ & $90+3 ?$ & 40,90 & 20 \\
\hline 12 & $\begin{array}{l}54 \text { (Guir Zil } \\
\text { Kabir) }\end{array}$ & $\begin{array}{l}\text { Unnamed Wadi / } \\
\text { Jaghjagh }\end{array}$ & 7 ha & $89+2 ?$ & $\mathrm{I} 2,7 \mathrm{I}$ & 35 \\
\hline I3 & 70 (Tell Badan) & $\begin{array}{l}\text { Jaghjagh, right } \\
\text { bank }\end{array}$ & I7,50 ha & $7 \mathrm{I}+5 ?$ & 4,05 & 49 \\
\hline I4 & $\begin{array}{l}44 \text { (Guir } \\
\text { Diouane) }\end{array}$ & $\begin{array}{l}\text { Unnamed Wadi / } \\
\text { Jaghjagh }\end{array}$ & o,8० ha & $7 \mathrm{I}+\mathrm{I} ?$ & 88,75 & 12 \\
\hline
\end{tabular}




\begin{tabular}{|c|c|c|c|c|c|c|}
\hline \multicolumn{2}{|c|}{$\begin{array}{l}\text { Ranking of sites according } \\
\text { to number of retained pot- } \\
\text { sherds }\end{array}$} & \multirow[t]{2}{*}{ Drainage Basin } & \multirow[t]{2}{*}{ Surface } & \multirow{2}{*}{$\begin{array}{l}\text { Number of } \\
\text { retained pot- } \\
\text { sherds from } \\
\text { the Old } \\
\text { Babylonian } \\
\text { Period } \\
\text { Total = } 3977 \\
+129 \text { ? }\end{array}$} & \multicolumn{2}{|c|}{$\begin{array}{l}\text { Ranking according to } \\
\text { population density }\end{array}$} \\
\hline Ranking & Site & & & & $\begin{array}{l}\text { No. of pot- } \\
\text { sherds/ha }\end{array}$ & Ranking \\
\hline I5 & 53 (Tell Kourdiss) & $\begin{array}{l}\text { Unnamed Wadi / } \\
\text { Jaghjagh }\end{array}$ & 2 ha & 66 & 33 & 24 \\
\hline 16 & 40 (Tell Qattine) & $\begin{array}{l}\text { Unnamed Wadi / } \\
\text { Dara }\end{array}$ & 0,50 ha & $56+\mathrm{I} ?$ & II2 & 7 \\
\hline I7 & $\begin{array}{l}\text { I8 (Tell el Ward } \\
\text { Charqi) }\end{array}$ & Wadi Zerkan & ca.r ha & $52+6 ?$ & 52 & I8 \\
\hline I8 & $\begin{array}{l}\text { I3 (Tell Aïn el } \\
\text { Abed) }\end{array}$ & $\begin{array}{l}\text { Wadi Zerkan / } \\
\text { spring }\end{array}$ & 0,50 ha & $47+2 ?$ & 94 & II \\
\hline 19 & 7 (Tell Qattine) & Khabur, right bank & 5 ha & $46+2 ?$ & 9,2 & 40 \\
\hline 20 & 56 (Tell Nurek) & Wadi Dara & 6 ha & $46+I ?$ & 7,6 & 44 \\
\hline $2 I$ & 28 (Tell Hassek) & $\begin{array}{l}\text { Unnamed Wadi/ } \\
\text { Aoueij, right bank }\end{array}$ & I, 50 ha & 45 & 30 & 25 \\
\hline 22 & $\begin{array}{l}4 \text { (Tell Umm el } \\
\text { Asafir) }\end{array}$ & Khabur, left bank & 0,50 ha & $3 I+2 ?$ & 62 & 16 \\
\hline 23 & $\begin{array}{l}23 \text { (Tell Aluq } \\
\text { Charqi) }\end{array}$ & Jirjirb Gharbi & O,Io ha & $30+I ?$ & 300 & 3 \\
\hline 24 & $\begin{array}{l}2 \text { (Tell Umm } \\
\text { Urhafa) }\end{array}$ & Khabur, left bank & I,5० ha & 30 & 20 & $3 I$ \\
\hline 25 & 26 (Tell Dabach) & $\begin{array}{l}\text { Wadi Seher / } \\
\text { Zerkan }\end{array}$ & 3,50 ha & $27+\mathrm{I} ?$ & $7,7 \mathrm{I}$ & 43 \\
\hline 26 & $\begin{array}{l}57 \text { (Tell Chil } \\
\text { Virane) }\end{array}$ & $\begin{array}{l}\text { Without wadi / } \\
\text { Dara }\end{array}$ & I,I5 ha & $24+4 ?$ & 20,86 & 30 \\
\hline 27 & $\begin{array}{l}\text { 5I (Tell Rhazal } \\
\text { Tahtani) }\end{array}$ & $\begin{array}{l}\text { Unnamed Wadi / } \\
\text { Zerkan }\end{array}$ & I,5० ha & $22+2 ?$ & 14,66 & 33 \\
\hline 28 & 39 (Tell Khaneke) & $\begin{array}{l}\text { Spring / Wadi } \\
\text { Dara }\end{array}$ & 0,20 ha & 22 & IIO & 8 \\
\hline 29 & 37 (Tell Bati) & $\begin{array}{l}\text { Wadi Rijla / } \\
\text { Jaghjagh }\end{array}$ & 5 ha & 22 & 4,4 & $4^{8}$ \\
\hline
\end{tabular}




\begin{tabular}{|c|c|c|c|c|c|c|}
\hline $\begin{array}{l}\text { Ranking o } \\
\text { to numbe } \\
\text { sherds }\end{array}$ & $\begin{array}{l}\text { f sites according } \\
r \text { of retained pot- }\end{array}$ & Drainage Basin & Surface & $\begin{array}{l}\text { Number of } \\
\text { retained pot- } \\
\text { sherds from }\end{array}$ & $\begin{array}{l}\text { Ranking acc } \\
\text { population }\end{array}$ & $\begin{array}{l}\text { ording to } \\
\text { lensity }\end{array}$ \\
\hline Ranking & Site & & & $\begin{array}{l}\text { Total }=3977 \\
+ \text { I29? }\end{array}$ & $\begin{array}{l}\text { No. of pot- } \\
\text { sherds/ha }\end{array}$ & Ranking \\
\hline 30 & $\begin{array}{l}29 \text { (Tell Aswad } \\
\text { Tahtani) }\end{array}$ & $\begin{array}{l}\text { Unnamed Wadi / } \\
\text { Jaghjagh }\end{array}$ & I ha & $2 \mathrm{I}+\mathrm{I} ?$ & $2 \mathrm{I}$ & 29 \\
\hline $3 I$ & I4 (Tell Jamilo) & $\begin{array}{l}\text { Wadi Aaoueij, left } \\
\text { bank }\end{array}$ & 8 ha & $20+2 ?$ & 2,5 & 52 \\
\hline 32 & I7 (Tell Effendi) & $\begin{array}{l}\text { Unnamed Wadi / } \\
\text { Jaghjagh }\end{array}$ & I,9० ha & I9 & IO & $38 a$ \\
\hline 33 & 58 (Tell Dukri) & $\begin{array}{l}\text { Unnamed Wadi / } \\
\text { Dara }\end{array}$ & 0,30 ha & $\mathrm{I} 8+\mathrm{I} ?$ & 60 & I7 \\
\hline 34 & 59 (Tell Jhach) & $\begin{array}{l}\text { Unnamed Wadi / } \\
\text { Dara }\end{array}$ & $0,5 \circ$ ha & $\mathrm{I} 8+\mathrm{I} ?$ & 36 & 23 \\
\hline 35 & $\begin{array}{l}60 \text { (Tell } \\
\text { Tcholama } \\
\text { Tahtani) }\end{array}$ & $\begin{array}{l}\text { Unnamed Wadi / } \\
\text { Dara }\end{array}$ & 6 ha & $17+3 ?$ & 2,83 & $5^{I}$ \\
\hline 36 & $\begin{array}{l}30 \text { (Tell Berguil } \\
\text { Buz) }\end{array}$ & $\begin{array}{l}\text { Unnamed Wadi / } \\
\text { Jaghjagh }\end{array}$ & 0,60 ha & I6 & 26,66 & 28 \\
\hline 37 & 20 (Tell Jamus) & Jirjib & 0,40 ha & $15+3 ?$ & 37,50 & 22 \\
\hline 38 & $\begin{array}{l}\text { 2I (Tell Barair } \\
\text { Kabir) }\end{array}$ & $\begin{array}{l}\text { Without Wadi / } \\
\text { Jirjib }\end{array}$ & 0,85 ha & $\mathrm{I} 5+\mathrm{I} ?$ & 17,64 & 32 \\
\hline 39 & $\begin{array}{l}63 \text { (Tell Abu } \\
\text { Hjeira 3) }\end{array}$ & $\begin{array}{l}\text { Wadi Awayj, right } \\
\text { bank }\end{array}$ & Unknown & $\mathrm{I} 4+\mathrm{I} ?$ & $?$ & $?$ \\
\hline 40 & 50 (Tell Mujarja) & Khabur, left bank & 0,50 ha & I4 & 28 & 27 \\
\hline $4 \mathrm{I}$ & 3 (Tell Tawil) & Khabur, left bank & I ha & $\mathrm{I} 2+4 ?$ & I2 & 36 \\
\hline $4^{2}$ & II (Tell Atchach) & Khabur, right bank & I ha & $\mathrm{II}+\mathrm{I} ?$ & II & 37 \\
\hline 43 & $\begin{array}{l}22 \text { (Tell ed } \\
\text { Daudiye) }\end{array}$ & $\begin{array}{l}\text { Without wadi / } \\
\text { Jirjib }\end{array}$ & I, 50 ha & $\mathrm{IO}+\mathrm{I} ?$ & 6,66 & 45 \\
\hline 44 & I (Tell Rommane) & Khabur, left bank & 2,50 ha & IO & 4 & 50 \\
\hline 45 & I5 (Tell Beydar) & $\begin{array}{l}\text { Wadi Aaoueij, } \\
\text { right bank }\end{array}$ & I 4,50 ha & $9+\mathrm{I} ?$ & 0,62 & 57 \\
\hline 46 & $\begin{array}{l}35 \text { (Tell Avguir } \\
\text { Fawqani) }\end{array}$ & $\begin{array}{l}\text { Unnamed Wadi / } \\
\text { Dara }\end{array}$ & 0,30 ha & $9+1 ?$ & 30 & $25 \mathrm{a}$ \\
\hline
\end{tabular}




\begin{tabular}{|c|c|c|c|c|c|c|}
\hline \multicolumn{2}{|c|}{$\begin{array}{l}\text { Ranking of sites according } \\
\text { to number of retained pot- } \\
\text { sherds }\end{array}$} & \multirow[t]{2}{*}{ Drainage Basin } & \multirow[t]{2}{*}{ Surface } & \multirow{2}{*}{$\begin{array}{l}\text { Number of } \\
\text { retained pot- } \\
\text { sherds from } \\
\text { the Old } \\
\text { Babylonian } \\
\text { Period } \\
\text { Total = } 3977 \\
+129 \text { ? }\end{array}$} & \multicolumn{2}{|c|}{$\begin{array}{l}\text { Ranking according to } \\
\text { population density }\end{array}$} \\
\hline Ranking & Site & & & & $\begin{array}{l}\text { No. of pot- } \\
\text { sherds/ha }\end{array}$ & Ranking \\
\hline 47 & $\begin{array}{l}5 \text { (Tell Asnane } \\
\text { Fawqani) }\end{array}$ & Khabur, left bank & I, $5 \circ$ ha & $8+2 ?$ & 5,33 & 46 \\
\hline 48 & I6 (Tell Khatun) & $\begin{array}{l}\text { Wadi Aoueij, left } \\
\text { bank }\end{array}$ & $\mathrm{I}, 20 \mathrm{ha}$ & $6+3 ?$ & 5 & 47 \\
\hline 49 & $\begin{array}{l}\text { I2 (Tell Ashnane } \\
\text { Charqi) }\end{array}$ & Wadi Zerkan & 0,75 ha & 6 & 8 & $4 \mathrm{I}$ \\
\hline 50 & II ter (unnamed) & Khabur, right bank & 0,07 ha & 5 & $7 \mathrm{I}, 42$ & I4 \\
\hline $5 I$ & 55 (Tell Dibe) & $\begin{array}{l}\text { Unnamed Wadi / } \\
\text { Jaghjagh }\end{array}$ & 0,50 ha & 5 & IO & 38 \\
\hline 52 & $\begin{array}{l}\text { I9 (Tell } \\
\text { Mohammad) }\end{array}$ & $\begin{array}{l}\text { Without Wadi / } \\
\text { Zerkan }\end{array}$ & o,Io ha & $4+I ?$ & 40 & $2 \mathrm{I}$ \\
\hline 53 & $\begin{array}{l}27 \text { (Tell ech } \\
\text { Chour Gharbi) }\end{array}$ & $\begin{array}{l}\text { Unnamed Wadi / } \\
\text { Zerkan }\end{array}$ & 6 ha & 4 & 0,66 & 56 \\
\hline 54 & $\begin{array}{l}48 \text { (without } \\
\text { name) }\end{array}$ & $\begin{array}{l}\text { Wadi Aaoueij, } \\
\text { right bank }\end{array}$ & $0,5 \circ$ ha & 4 & 8 & $4^{\mathrm{Ia}}$ \\
\hline 55 & $\begin{array}{l}47 \text { (Tell Abu } \\
\text { Hufur modern) }\end{array}$ & $\begin{array}{l}\text { Wadi Aoueij, right } \\
\text { bank }\end{array}$ & 2,60 ha & 3 & $\mathrm{I}, \mathrm{I} 5$ & 54 \\
\hline 56 & 52 (Tell Bughaz) & $\begin{array}{l}\text { "sea" / Wadi } \\
\text { Zerkan }\end{array}$ & O,I5 ha & $2+\mathrm{I} ?$ & $\mathrm{I} 3,33$ & 34 \\
\hline 57 & 6 (Tell Fakhariya) & Khabur, left bank & $3,5 \circ \mathrm{ha}$ & 2 & 0,57 & 58 \\
\hline 58 & 8 (Tell Majdel) & Khabur, right bank & 25 ha & 2 & 0,08 & 59 \\
\hline 59 & 36 (Tell Fatme) & $\begin{array}{l}\text { Unnamed / } \\
\text { Jaghjagh }\end{array}$ & 0,50 ha & $I+I ?$ & 2 & 53 \\
\hline 60 & 6I (Tell Ghara) & Khabur, left bank & I ha & I & I & 55 \\
\hline
\end{tabular}


A.2 The Late Khabur and/or Early Mittani Periods (Table II and Fig.1)

Based on a comparison with the material from Muhammad Diyab VIII and other sites, certain pottery types, classified by shape and decoration and amounting to 2I series (eight of which we are still uncertain about ${ }^{2}$ ) based on 46 potsherds, were attributed to this period. 33

The fact that there are six times fewer diagnostic series in this period than in the previous one cannot be assumed to correctly reflect this phase of settlement. In addition, as mentioned above, the majority of earlier series continued over the course of this phase. As a result, the numbers obtained are insufficient to allow us to make an estimate of the size of the population in this phase, and can only confirm which sites were in fact inhabited during that time.

More than a third of the retained sherds come from T.38 (I4 sherds); the next largest group comes from T. 45 ( 6 sherds) and T. 3 I gives us only 4 sherds, of which 3 are uncertain. T. 7, 23, 24, 25, 32, and 42 each yielded two potsherds from this period. Finally, the sites T. I, 2, I3, I4, I6, 28, 34, 4I, 43, 44, 53, 54 and 58 each yielded only one potsherd from this phase.

Our image regarding the population of this period does not suggest major changes to the preceding period; for the most part we see the same sites organized in the same ways.

If we take not only the total number of retained potsherds into consideration but also the surface area of the tells, we observe that seven of the most densely populated sites (right column) covered less than I.5 ha each (T. 23, 43, 58, 32, 25, I3, 44), two (T. 38 and 24) had a surface area between I. 5 and 4 ha, and one (T. 45 ) was over 4 ha. The majority of them were already densely populated during the previous period and the map indicating the distribution of the population among sites barely changes (Fig. I).

32 Faivre I999, vol. I, 237.

33 Faivre I999, vol. 2, fig. I47. 
Table II | List of sites that yielded potsherds dating to the late Khabur and/or early Mittani period, taken from the sample retained after the first selection. They are sorted according to the quantity of sherds retained (left column) and by population density (right column). Potsherds followed by a question mark were not counted.

\begin{tabular}{|c|c|c|c|c|c|c|}
\hline \multicolumn{2}{|c|}{$\begin{array}{l}\text { Ranking of sites according } \\
\text { to number of retained pot- } \\
\text { sherds }\end{array}$} & \multirow[t]{2}{*}{ Drainage Basin } & \multirow[t]{2}{*}{ Surface } & \multirow{2}{*}{\begin{tabular}{|l} 
Number of \\
retained pot- \\
sherds of the \\
later Khabur \\
and/or the \\
beginning of \\
the Mittani \\
Period \\
\\
Total = \\
$46+3 ?$
\end{tabular}} & \multicolumn{2}{|c|}{$\begin{array}{l}\text { Ranking according to } \\
\text { population density }\end{array}$} \\
\hline Ranking & Site & & & & $\begin{array}{l}\text { No. of pot- } \\
\text { sherds/ha }\end{array}$ & Ranking \\
\hline $\mathbf{I}$ & $\begin{array}{l}38 \text { (Tell Aïn el } \\
\text { Qurd) }\end{array}$ & Wadi Dara & 2,42 ha & I4 & 5,6 & 2 \\
\hline 2 & 45 (Tell Ahmar) & $\begin{array}{l}\text { Unnamed Wadi / } \\
\text { Jaghjagh }\end{array}$ & $4,9 \circ$ ha & 6 & $\mathrm{I}, 2$ & 9 \\
\hline 3 & 3I (Tell Dibak) & $\begin{array}{l}\text { Wadi Aoueij, left } \\
\text { bank }\end{array}$ & ca. 2,30 ha & $I+3 ?$ & 0,43 & I7 \\
\hline 4 & 7 (Tell Qattine) & Khabur, right bank & 5 ha & 2 & 0,4 & 18 \\
\hline 5 & $\begin{array}{l}23 \text { (Tell Aluq } \\
\text { Charqi) }\end{array}$ & Jirjirb Gharbi & O,Io ha & 2 & 20 & $\mathbf{I}$ \\
\hline 6 & 24 (Tell Hermel) & Wadi Zerkan & 2 ha & 2 & I & IO \\
\hline 7 & 25 (Tell Arade) & Wadi Zerkan & I ha & 2 & 2 & 6 \\
\hline 8 & 32 (Tell Kdich) & $\begin{array}{l}\text { Wadi Aoueij, left } \\
\text { bank }\end{array}$ & o,8० ha & 2 & 2,5 & 5 \\
\hline 9 & 42 (Tell Arbid) & $\begin{array}{l}\text { Unnamed Wadi / } \\
\text { Jaghjagh }\end{array}$ & 7 ha & 2 & 0,28 & 20 \\
\hline I0 & I (Tell Rommane) & Khabur, left bank & $2,5 \circ$ ha & I & 0,4 & I8a \\
\hline II & $\begin{array}{l}2 \text { (Tell Umm } \\
\text { Urhafa) }\end{array}$ & Khabur, left bank & $\mathrm{I}, 50 \mathrm{ha}$ & I & 0,66 & 13 \\
\hline 12 & $\begin{array}{l}\text { I3 (Tell Aïn el } \\
\text { Abed) }\end{array}$ & $\begin{array}{l}\text { Wadi Zerkan / } \\
\text { spring }\end{array}$ & 0,50 ha & I & 2 & $6 a$ \\
\hline 13 & I4 (Tell Jamilo) & $\begin{array}{l}\text { Wadi Aoueij, left } \\
\text { bank }\end{array}$ & 8 ha & I & $0, \mathrm{I2}$ & 22 \\
\hline
\end{tabular}




\begin{tabular}{|c|c|c|c|c|c|c|}
\hline \multicolumn{2}{|c|}{$\begin{array}{l}\text { Ranking of sites according } \\
\text { to number of retained pot- } \\
\text { sherds }\end{array}$} & \multirow[t]{2}{*}{ Drainage Basin } & \multirow[t]{2}{*}{ Surface } & \multirow{2}{*}{\begin{tabular}{|l|} 
Number of \\
retained pot- \\
sherds of the \\
later Khabur \\
and/or the \\
beginning of \\
the Mittani \\
Period \\
\\
Total = \\
$46+3 ?$
\end{tabular}} & \multicolumn{2}{|c|}{$\begin{array}{l}\text { Ranking according to } \\
\text { population density }\end{array}$} \\
\hline Ranking & Site & & & & $\begin{array}{l}\text { No. of pot- } \\
\text { sherds/ha }\end{array}$ & Ranking \\
\hline 14 & I6 (Tell Khatun) & $\begin{array}{l}\text { Wadi Aoueij, left } \\
\text { bank }\end{array}$ & I, 20 ha & I & 0,83 & II \\
\hline 15 & 28 (Tell Hassek) & $\begin{array}{l}\text { Unnamed Wadi } \\
\text { /Aoueij, right bank }\end{array}$ & I,50 ha & I & 0,66 & Iзa \\
\hline 16 & 34 (Tell Atach) & $\begin{array}{l}\text { Unnamed Wadi / } \\
\text { Jaghjagh }\end{array}$ & I,20 ha & I & 0,83 & IIa \\
\hline 17 & 4I (Tell Baqar) & $\begin{array}{l}\text { Unnamed Wadi } \\
\text { /Dara }\end{array}$ & 2,20 ha & I & 0,45 & 16 \\
\hline 18 & 43 (Tell Farho) & Wadi Dara & ca. $0,3 \circ$ ha & I & 3,33 & 3 \\
\hline 19 & $\begin{array}{l}44 \text { (Guir } \\
\text { Diouane) }\end{array}$ & $\begin{array}{l}\text { Unnamed Wadi / } \\
\text { Jaghjagh }\end{array}$ & o,8० ha & I & $\mathrm{I}, 25$ & 8 \\
\hline 20 & 53 (Tell Kourdiss) & $\begin{array}{l}\text { Unnamed Wadi / } \\
\text { Jaghjagh }\end{array}$ & 2 ha & I & 0,5 & 15 \\
\hline $2 \mathrm{I}$ & $\begin{array}{l}54 \text { (Tell Guire Zil } \\
\text { Kebir) }\end{array}$ & $\begin{array}{l}\text { Unnamed Wadi / } \\
\text { Jaghjagh }\end{array}$ & 7 ha & I & $\mathrm{O}, \mathrm{I} 4$ & $2 \mathrm{I}$ \\
\hline 22 & 58 (Tell Dukri) & $\begin{array}{l}\text { Unnamed Wadi / } \\
\text { Dara }\end{array}$ & 0,30 ha & I & 3,33 & $3 a$ \\
\hline
\end{tabular}




\section{A.3 The Mittani Period (Table III and Fig. 2)}

The pottery of this period was defined by comparison to the material from Muhammad Diyab VII and other sites. There are I33 series, I4 of which are uncertain, 34 based upon more than 2,000 of the retained potsherds $(1987+\mathrm{I} 35$ ?). This is slightly less than the number of diagnostic series used for the Old Babylonian period while the number of potsherds retained is roughly half as much. Nevertheless, this sample seems sufficient for making a comparison between the Mittani and the Old Babylonian periods.

Table III shows that almost as many sites are occupied in the Mittani period as during the preceding period, but almost one third of the potsherds come from five sites, where from Ioo to 200 potsherds have been retained (T. 38, 45, 28, 42, and 32). Eight other sites delivered between 50 and Ioo potsherds (T. 3I, 24, 33, 34, 70, 2, 54 and 4); twenty sites yielded between ca. 20 and 40 ; and 25 other sites provided between I and 15 potsherds of this period.

If we examine the relationship between the number of potsherds in this period and the surface area of the tell, we observe that eight of the ten most densely populated sites are less than I.5 ha (T. 32, 4, 43, 34, I3, I9, 44, 40), while two have a surface area of between I.5 and 4 ha (T. 28 and 38). Three of the sites that are the most densely populated during the Mittani period were not settled in the earlier periods: T. 4, I9, and 28, while another two, T. I3 and 44, appeared only at the end of the late Khabur/early Mittani period. We therefore distinctly see a change in the settlement pattern of the region, most clearly with the emergence of a distinct settlement in the western zone, especially along the Khabur itself (Fig. 2). 
Table III | List of sites that yielded potsherds dating to the Mittani period, taken from the material retained after the first selection. They are ordered according to the quantity of potsherds retained (left column) and by population density (right column). Potsherds followed by a question mark were not counted.

\begin{tabular}{|c|c|c|c|c|c|c|}
\hline \multicolumn{2}{|c|}{$\begin{array}{l}\text { Ranking of sites according } \\
\text { to number of retained pot- } \\
\text { sherds }\end{array}$} & \multirow[t]{2}{*}{ Drainage Basin } & \multirow[t]{2}{*}{ Surface } & \multirow{2}{*}{$\begin{array}{l}\text { Number of } \\
\text { retained pot- } \\
\text { sherds of the } \\
\text { Mittani } \\
\text { Period } \\
\text { Total = I987 } \\
+ \text { I35? }\end{array}$} & \multicolumn{2}{|c|}{$\begin{array}{l}\text { Ranking according to } \\
\text { population density }\end{array}$} \\
\hline Ranking & Site & & & & $\begin{array}{l}\text { No. of pot- } \\
\text { sherds/ha }\end{array}$ & Ranking \\
\hline I & $\begin{array}{l}38 \text { (Tell Aïn el } \\
\text { Qurd) }\end{array}$ & Wadi Dara & & $196+6 ?$ & 78,4 & 5 \\
\hline 2 & 45 (Tell Ahmar) & $\begin{array}{l}\text { Unnamed Wadi/ } \\
\text { Jaghjagh }\end{array}$ & $4,9 \circ$ ha & $165+15 ?$ & 33 & 19 \\
\hline 3 & 28 (Tell Hassek) & $\begin{array}{l}\text { Unnamed Wadi/ } \\
\text { Aoueij, right bank }\end{array}$ & I, 50 ha & $\mathrm{I} 59+\mathrm{I} ?$ & 106 & 3 \\
\hline 4 & 42 (Tell Arbid) & $\begin{array}{l}\text { Unnamed Wadi/ } \\
\text { Jaghjagh }\end{array}$ & 7 ha & $\mathrm{II} 2+\mathrm{I} 6 ?$ & I6 & 26 \\
\hline $5^{\mathrm{e}-}$ & 32 (Tell Kdich) & Aoueij, left bank & o,8o ha & $98+5 ?$ & 122,5 & $\mathbf{I}$ \\
\hline 6 & 3I (Tell Dibak) & Aoueij, left bank & ca. 2,30 ha & $78+7 ?$ & 33,91 & I8 \\
\hline 7 & 24 (Tell Hermel) & Wadi Zerkan & 2 ha & $75+6 ?$ & 37,5 & 16 \\
\hline 8 & 33 (Tell Hanoua) & Aoueij, left bank & 3,90 ha & $68+2 ?$ & I7 & 25 \\
\hline 9 & 34 (Tell Atach) & $\begin{array}{l}\text { Unnamed Wadi/ } \\
\text { Jaghjagh }\end{array}$ & I,20 ha & $65+6 ?$ & 54,16 & 6 \\
\hline I0 & 70 (Tell Badan) & $\begin{array}{l}\text { Jaghjagh, right } \\
\text { bank }\end{array}$ & I7, 50 ha & $63+13 ?$ & 3,6 & 45 \\
\hline II & $\begin{array}{l}2 \text { (Tell Umm } \\
\text { Urhafa) }\end{array}$ & Khabur & I, 50 ha & $60+1 ?$ & 40 & 12 \\
\hline 12 & $\begin{array}{l}54 \text { (Tell Guire Zil } \\
\text { Kebir) }\end{array}$ & $\begin{array}{l}\text { Unnamed Wadi/ } \\
\text { Jaghjagh }\end{array}$ & 7 ha & $58+2 ?$ & 8,28 & 33 \\
\hline 13 & $\begin{array}{l}4 \text { (Tell Umm el } \\
\text { Asafir) }\end{array}$ & Khabur, left bank & 0,50 ha & $54+4 ?$ & I08 & 2 \\
\hline I4 & 37 (Tell Bati) & Rijla / Jaghjagh & 5 ha & $4 I+2 ?$ & 8,2 & 34 \\
\hline 15 & 25 (Tell Arade) & Wadi Zerkan & I ha & $4 \mathrm{I}$ & $4 \mathrm{I}$ & II \\
\hline 16 & $\begin{array}{l}57 \text { (Tell Chil } \\
\text { Virane) }\end{array}$ & $\begin{array}{l}\text { Without wadi / } \\
\text { Dara }\end{array}$ & I,I5 ha & $40+I ?$ & 34,78 & I7 \\
\hline 17 & $\begin{array}{l}\text { 5I (Tell Rhazal } \\
\text { Tahtani) }\end{array}$ & $\begin{array}{l}\text { Unnamed / } \\
\text { Zerkan }\end{array}$ & I, 50 ha & $39+3 ?$ & 26 & 22 \\
\hline I8 & $\begin{array}{l}44 \text { (Guir } \\
\text { Diouane) }\end{array}$ & $\begin{array}{l}\text { Unnamed wadi / } \\
\text { Jaghjagh }\end{array}$ & o,8० ha & $37+5 ?$ & 46,25 & 9 \\
\hline
\end{tabular}




\begin{tabular}{|c|c|c|c|c|c|c|}
\hline \multicolumn{2}{|c|}{$\begin{array}{l}\text { Ranking of sites according } \\
\text { to number of retained pot- } \\
\text { sherds }\end{array}$} & \multirow[t]{2}{*}{ Drainage Basin } & \multirow[t]{2}{*}{ Surface } & \multirow{2}{*}{$\begin{array}{l}\text { Number of } \\
\text { retained pot- } \\
\text { sherds of the } \\
\text { Mittani } \\
\text { Period } \\
\text { Total = I98 } 87 \\
+ \text { I35? }\end{array}$} & \multicolumn{2}{|c|}{$\begin{array}{l}\text { Ranking according to } \\
\text { population density }\end{array}$} \\
\hline Ranking & Site & & & & $\begin{array}{l}\text { No. of pot- } \\
\text { sherds/ha }\end{array}$ & Ranking \\
\hline 19 & I4 (Tell Jamilo) & Aoueij & 8 ha & $37+2 ?$ & 4,62 & 44 \\
\hline 20 & $\begin{array}{l}\text { I8 (Tell el Ward } \\
\text { Charqi) }\end{array}$ & Wadi Zerkan & ca. I ha & $33+3 ?$ & 33 & I9a \\
\hline $2 I$ & 26 (Tell Dabach) & Seher/Zerkan & $3,5 \circ \mathrm{ha}$ & 30 & 8,57 & 32 \\
\hline 22 & 7 (Tell Qattine) & Khabur & 5 ha & $29+4 ?$ & 5,8 & $4 \mathrm{I}$ \\
\hline 23 & I5 (Tell Beydar) & Aoueij & $\mathrm{I} 4,50 \mathrm{ha}$ & $29+3 ?$ & 2 & 50 \\
\hline 24 & 43 (Tell Farho) & Wadi Dara & ca. $0,3 \circ$ ha & 27 & 90 & 4 \\
\hline 25 & $\begin{array}{l}\text { I3 (Tell Aïn el } \\
\text { Abed) }\end{array}$ & $\begin{array}{l}\text { Wadi Zerkan/ } \\
\text { spring }\end{array}$ & 0,50 ha & $26+I ?$ & 52 & 7 \\
\hline 26 & 53 (Tell Kourdiss) & $\begin{array}{l}\text { Unnamed Wadi / } \\
\text { Jaghjagh }\end{array}$ & 2 ha & $25+I ?$ & $\mathrm{I} 2,5$ & 29 \\
\hline 27 & I7 (Tell Effendi) & $\begin{array}{l}\text { Unnamed / } \\
\text { Jaghjagh }\end{array}$ & $\mathrm{I}, 9 \circ \mathrm{ha}$ & 25 & $\mathrm{I} 3, \mathrm{I} 5$ & 27 \\
\hline 28 & $\begin{array}{l}63 \text { (Tell Abu } \\
\text { Hjeira 3) }\end{array}$ & Aoueij & Unknown & 25 & $?$ & $?$ \\
\hline 29 & $\begin{array}{l}30 \text { (Tell Berguil } \\
\text { Buz) }\end{array}$ & $\begin{array}{l}\text { Unnamed / } \\
\text { Jaghjagh }\end{array}$ & $0,6 \circ$ ha & $24+2 ?$ & 40 & I2a \\
\hline 30 & $\begin{array}{l}40 \text { (Tell } \\
\text { Kourdiss) }\end{array}$ & $\begin{array}{l}\text { Unnamed } \\
\text { Wadi/Dara }\end{array}$ & 0,50 ha & $23+I ?$ & 46 & IO \\
\hline $3 \mathbf{I}$ & 4I (Tell Baqar) & $\begin{array}{l}\text { Unnamed Wadi / } \\
\text { Dara }\end{array}$ & 2,20 ha & $2 I+3 ?$ & 9,54 & $3 \mathbf{I}$ \\
\hline 32 & $\begin{array}{l}60 \text { (Tell Tcholama } \\
\text { Tahtani) }\end{array}$ & Unnamed / Dara & 6 ha & $2 \mathrm{I}$ & 3,5 & 46 \\
\hline 33 & 50 (Tell Mujarja) & Khabur & 0,50 ha & $19+2 ?$ & 38 & 15 \\
\hline 34 & $\begin{array}{l}27 \text { (Tell ech } \\
\text { Chour Gharbi) }\end{array}$ & $\begin{array}{l}\text { Unnamed / } \\
\text { Zerkan }\end{array}$ & 6 ha & $\mathrm{I} 4$ & 2,33 & 49 \\
\hline 35 & I (Tell Rommane) & Khabur & $2,5 \circ \mathrm{ha}$ & $\mathrm{I} 2+\mathrm{I} ?$ & 4,8 & $4^{2}$ \\
\hline 36 & $\begin{array}{l}\text { 2I (Tell Barair } \\
\text { Kabir) }\end{array}$ & $\begin{array}{l}\text { Without wadi / } \\
\text { Jirjib }\end{array}$ & 0,85 ha & II & $\mathrm{I} 2,94$ & 28 \\
\hline 37 & $\begin{array}{l}35 \text { (Tell Avguir } \\
\text { Fawqani) }\end{array}$ & Unnamed / Dara & 0,30 ha & $8+2 ?$ & 26,66 & $2 \mathrm{I}$ \\
\hline 38 & 20 (Tell Jamus) & Jirjib & 0,40 ha & $8+\mathrm{I} ?$ & 20 & 23 \\
\hline
\end{tabular}




\begin{tabular}{|c|c|c|c|c|c|c|}
\hline \multicolumn{2}{|c|}{$\begin{array}{l}\text { Ranking of sites according } \\
\text { to number of retained pot- } \\
\text { sherds }\end{array}$} & \multirow[t]{2}{*}{ Drainage Basin } & \multirow[t]{2}{*}{ Surface } & \multirow{2}{*}{$\begin{array}{l}\begin{array}{l}\text { Number of } \\
\text { retained pot- } \\
\text { sherds of the } \\
\text { Mittani } \\
\text { Period } \\
\text { Total }=1987 \\
+\mathrm{I} 35 ?\end{array}\end{array}$} & \multicolumn{2}{|c|}{$\begin{array}{l}\text { Ranking according to } \\
\text { population density }\end{array}$} \\
\hline Ranking & Site & & & & $\begin{array}{l}\text { No. of pot- } \\
\text { sherds/ha }\end{array}$ & Ranking \\
\hline 39 & $\begin{array}{l}29 \text { (Tell Aswad } \\
\text { Tahtani) }\end{array}$ & $\begin{array}{l}\text { Unnamed / } \\
\text { Jaghjagh }\end{array}$ & I ha & 8 & 8 & 35 \\
\hline 40 & $\begin{array}{l}22 \text { (Tell ed } \\
\text { Daudiye) }\end{array}$ & $\begin{array}{l}\text { Without wadi / } \\
\text { Jirjib }\end{array}$ & I, 50 ha & $7+1 ?$ & 4,66 & 43 \\
\hline $4 \mathrm{I}$ & ${ }_{5}^{6}$ (Tell Qattine) & Wadi Dara & 6 ha & $7+\mathrm{I} ?$ & $\mathrm{I}, \mathrm{I} 6$ & 55 \\
\hline 42 & 3 (Tell Tawil) & Khabur & I ha & 7 & 7 & 37 \\
\hline 43 & 59 (Tell Jhach) & Unnamed / Dara & 0,50 ha & $6+2 ?$ & I2 & 30 \\
\hline 44 & II (Tell Atchach) & Khabur & I ha & $6+\mathrm{I} ?$ & 6 & 39 \\
\hline 45 & 8 (Tell Majdel) & Khabur & 25 ha & 6 & 0,24 & 57 \\
\hline 46 & $\begin{array}{l}\text { I2 (Tell Ashnane } \\
\text { Charqi) }\end{array}$ & Zerkan & 0,75 ha & 6 & 8 & $35^{\mathrm{a}}$ \\
\hline 47 & 6 (Tell Fakhariya) & Khabur & 3,50 ha & 5 & $\mathrm{I}, 42$ & 54 \\
\hline $4^{8}$ & $\begin{array}{l}\text { I9 (Tell } \\
\text { Mohammad) }\end{array}$ & $\begin{array}{l}\text { Without wadi / } \\
\text { Zerkan }\end{array}$ & o, Io ha & 5 & 50 & 8 \\
\hline 49 & $\begin{array}{l}47 \text { (Tell Abu } \\
\text { Hufur modern) }\end{array}$ & Aoueij & $2,6 \circ$ ha & 5 & $I, 9^{2}$ & 53 \\
\hline 50 & $\begin{array}{l}5 \text { (Tell Asnane } \\
\text { Fawqani) }\end{array}$ & Khabur & I, $5 \circ$ ha & $4+2 ?$ & 2,66 & 47 \\
\hline $5^{I}$ & 39 (Tell Khaneke) & Source / Dara & 0,20 ha & 4 & 20 & $23 a$ \\
\hline 52 & $\begin{array}{l}23 \text { (Tell Aluq } \\
\text { Charqi) }\end{array}$ & Jirjirb Gharbi & o, Io ha & 4 & 40 & I2b \\
\hline 53 & I6 (Tell Khatun) & Aoueij & I, 20 ha & 3 & 2,5 & $4^{8}$ \\
\hline 54 & 48 (unnamed) & Aoueij & $0,5 \circ$ ha & 3 & 6 & $39 a$ \\
\hline 55 & 58 (Tell Dukri) & Unnamed / Dara & 0,30 ha & 2 & 6,66 & 38 \\
\hline 56 & 36 (Tell Fatme) & $\begin{array}{l}\text { Unnamed / } \\
\text { Jaghjagh }\end{array}$ & 0,50 ha & $I+I ?$ & 2 & $50 a$ \\
\hline 57 & 55 (Tell Dibe) & $\begin{array}{l}\text { Unnamed / } \\
\text { Jaghjagh }\end{array}$ & $0,5 \circ \mathrm{ha}$ & $I+I ?$ & 2 & $50 \mathrm{~b}$ \\
\hline 58 & Io (Tell Tawila) & Khabur & I, $6 \circ$ ha & I & 0,62 & 56 \\
\hline
\end{tabular}




\section{B Study based on the material initially collected during the survey35}

It is unfortunate that there is no possibility to directly compare the material initially collected with the data presented above, as they are based on different typological and chronological analyses. For example, in our notes on the initial material, the intermediary period, mentioned here as 'late Khabur/early Mittani', had not yet been identified. Overall, also, the typology for the general collection is less complex. In addition, I do not wish to claim that this article definitively identifies the chronological affiliations of each ceramic series; instead, the work presented below is based on my general impressions of the material as it was initially surveyed on each site. This approach is certainly less precise than the method described above, but I believe that it nevertheless allows us to better glean general tendencies. In the end, we will see that this method yielded results similar to Faivre's work.

\section{B.1 The Khabur Period (Table IV and Fig. 1)}

We see first of all that the number of sites which yielded material from this period is limited -24 of the 63 surveyed. None of those identified as having a limited number of potsherds in the preceeding study appears here. Next we notice that the quantity of potsherds collected varies greatly by site, from more than $\mathrm{I}, 000$ to only 7 potsherds. A hierarchy of sites is then evident, formed neatly into discrete groups: one site, Tall Dibak (3I) on the wadi Aaoueij, stands in a category by itself with more than I,००० potsherds collected for this period. Another three sites each yielded roughly 500 potsherds (T. 32, 33, and 42). Four sites produced between 200 and 300 (T. 45, 38, 25, 43), and finally a group of four much smaller sites each furnished between 70 and Ioo potsherds (T. 53, 24, 34, 4I). The last twelve sites each produced less than 50 potsherds.

Although the ordering among the largest sites is here sometimes slightly different than that proposed by Faivre in his analysis, we nevertheless see that the sites are the same. The absence of Khabur-period potsherds along the river is however much more evident here, as only T. 7 yielded any at all.

Certain sites were unoccupied except on a small part of the tell, ${ }^{6}$ a fact which is not available in the earlier study. This has important consequences for our understanding of the population density of a site, and therefore for its classification. Once again, the order is slightly modified in relation to what was proposed earlier, but the changes are relatively small. The two most densely populated sites (T. 32 and 43) are both less than I ha. Only T. 3 I

35 Here we only counted the potsherds that were diagnostic in either form or decoration but not the shapeless potsherds.

36 In these cases, the area for which an occupation is attested is indicated by a capital letter in the column of the number of potsherds collected. 
and 33, which are geographically quite close to each other, ranked third and sixth respectively for population density. Although they have a larger surface area, they are still relatively small (2.3 and 3.9 ha). The tells of larger dimensions (larger than 5 ha), do not appear before number $\mathrm{I} 3$ on the table (T. 42, 45, 56, 54), which seems to indicate that, despite their considerable geographic size, they were not very densely populated at this time.

Table IV | List of sites, which yielded potsherds dating to the "Khabur period", based on analysis of all potsherds originally collected. Sites are ordered by quantity of potsherds collected (left column) and by order of density measured as a ratio of quantity to surface (right column).

\begin{tabular}{|c|c|c|c|c|c|c|}
\hline \multicolumn{2}{|c|}{$\begin{array}{l}\text { Ranking of sites according } \\
\text { to number of collected pot- } \\
\text { sherds }\end{array}$} & \multirow[t]{2}{*}{ Drainage Basin } & \multirow[t]{2}{*}{ Surface } & \multirow{2}{*}{$\begin{array}{l}\begin{array}{l}\text { Number of } \\
\text { collected } \\
\text { potsherds of } \\
\text { the Khabur } \\
\text { Period }\end{array} \\
\text { Total }=4517\end{array}$} & \multicolumn{2}{|c|}{$\begin{array}{l}\text { Ranking according to } \\
\text { population density }\end{array}$} \\
\hline Ranking & Site & & & & $\begin{array}{l}\text { Number of } \\
\text { potsherds / } \\
\text { ha }\end{array}$ & Ranking \\
\hline $\mathbf{I}$ & 3I (Tell Dibak) & Aoueij, left bank & ca. 2,30 ha & II99 & 521,30 & 3 \\
\hline 2 & 32 (Tell Kdich) & Aoueij, left bank & $0,8 \circ$ ha & 586 & 732,5 & $\mathbf{I}$ \\
\hline 3 & 33 (Tell Hanoua) & Aoueij, left bank & ca. 3,90 ha & 580 & I4 $8,7 \mathrm{I}$ & 6 \\
\hline 4 & 42 (Tell Arbid) & $\begin{array}{l}\text { Unnamed Wadi / } \\
\text { Jaghjagh }\end{array}$ & 7 ha & 493 & 70,42 & I3 \\
\hline 5 & 45 (Tell Ahmar) & $\begin{array}{l}\text { Unnamed Wadi / } \\
\text { Jaghjagh }\end{array}$ & $4,9 \circ$ ha & 284 & 57,95 & I4 \\
\hline 6 & $\begin{array}{l}38 \text { (Tell Aïn el } \\
\text { Qurd) }\end{array}$ & Wadi Dara & 2,42 ha & 276 & II 4,04 & 8 \\
\hline 7 & 25 (Tell Arade) & Wadi Zerkan & I ha & 230 & 230 & 5 \\
\hline 8 & 43 (Tell Farho) & Wadi Dara & ca. $0,3 \circ$ ha & 210 & 700 & 2 \\
\hline 9 & 53 (Tell Kourdiss) & $\begin{array}{l}\text { Unnamed Wadi / } \\
\text { Jaghjagh }\end{array}$ & 2 ha & 99 & 49,5 & 16 \\
\hline IO & 24 (Tell Hermel) & Wadi Zerkan & 2 ha & 95 & 47,5 & I7 \\
\hline II & 34 (Tell Atach) & $\begin{array}{l}\text { Unnamed Wadi / } \\
\text { Jaghjagh }\end{array}$ & I, 20 ha & 86 & $7 \mathrm{I}, 66$ & I2 \\
\hline 12 & 4I (Tell Baqar) & $\begin{array}{l}\text { Unnamed Wadi / } \\
\text { Dara }\end{array}$ & $2,20 \mathrm{ha}$ & 72 & 32,72 & I9 \\
\hline I3 & 40 (Tell Qattine) & $\begin{array}{l}\text { Unnamed Wadi / } \\
\text { Dara }\end{array}$ & 0,50 ha & 46 & 92 & IO \\
\hline
\end{tabular}




\begin{tabular}{|c|c|c|c|c|c|c|}
\hline \multicolumn{2}{|c|}{$\begin{array}{l}\text { Ranking of sites according } \\
\text { to number of collected pot- } \\
\text { sherds }\end{array}$} & \multirow[t]{2}{*}{ Drainage Basin } & \multirow[t]{2}{*}{ Surface } & \multirow{2}{*}{$\begin{array}{l}\begin{array}{l}\text { Number of } \\
\text { collected } \\
\text { potsherds of } \\
\text { the Khabur } \\
\text { Period }\end{array} \\
\text { Total }=4517\end{array}$} & \multicolumn{2}{|c|}{$\begin{array}{l}\text { Ranking according to } \\
\text { population density }\end{array}$} \\
\hline Ranking & Site & & & & $\begin{array}{l}\text { Number of } \\
\text { potsherds/ } \\
\text { ha }\end{array}$ & Ranking \\
\hline 14 & $\begin{array}{l}44 \text { (Guir } \\
\text { Diouane) }\end{array}$ & $\begin{array}{l}\text { Unnamed Wadi / } \\
\text { Jaghjagh }\end{array}$ & o,8० ha & 43 & 53,75 & I5 \\
\hline $15 \mathrm{e}$ & ${ }_{5} 6$ (Tell Nurek) & Wadi Dara & 6 ha & $4 \mathrm{I}$ & 6,83 & $2 \mathrm{I}$ \\
\hline 16 & 58 (Tell Dukri) & $\begin{array}{l}\text { Unnamed Wadi / } \\
\text { Dara }\end{array}$ & $\begin{array}{l}0,30 \text { ha } \\
\text { but A only } \\
=0,20 \text { ha }\end{array}$ & 29 (A) & I45 & 7 \\
\hline 17 & $\begin{array}{l}23 \text { (Tell Aluq } \\
\text { Charqi) }\end{array}$ & Jirjib Gharbi & O,Io ha & 25 & 250 & 4 \\
\hline 18 & $\begin{array}{l}54 \text { (Guir Zil } \\
\text { Kabir) }\end{array}$ & $\begin{array}{l}\text { Unnamed Wadi / } \\
\text { Jaghjagh }\end{array}$ & 7 ha & 24 & 3,42 & 22 \\
\hline 19 & $\begin{array}{l}\text { I } 8 \text { (Tell el Ward } \\
\text { Charqi) }\end{array}$ & Wadi Zerkan & ca. I ha & 22 & 22 & 20 \\
\hline 20 & 20 (Tell Jamus) & Jirjib & $\begin{array}{l}\circ, 40 \text { ha } \\
\text { but A only } \\
=0,20 \text { ha }\end{array}$ & $20(\mathrm{~A})$ & 100 & 9 \\
\hline $2 I$ & $\begin{array}{l}\text { I3 (Tell Aïn el } \\
\text { Abed) }\end{array}$ & $\begin{array}{l}\text { Wadi Zerkan / } \\
\text { spring }\end{array}$ & o,50 ha & 20 & 40 & I8 \\
\hline 22 & 7 (Tell Qattine) & Khabur, right bank & $\begin{array}{l}5 \text { ha }-0,30 \\
(B)=4,70 \\
\text { ha }\end{array}$ & I5 (A, C) & 3,19 & 23 \\
\hline 23 & $\begin{array}{l}29 \text { (Tell Aswad } \\
\text { Tahtani) }\end{array}$ & $\begin{array}{l}\text { Unnamed Wadi / } \\
\text { Jaghjagh }\end{array}$ & $\begin{array}{l}\text { I ha but A } \\
\text { only }= \\
\text { o,I } 8 \text { ha }\end{array}$ & I5 (A) & 83,33 & II \\
\hline 24 & I4 (Tell Jamilo) & Aoueij, left bank & $\begin{array}{l}8 \text { ha, but } A \\
\text { only }=3 \text { ha }\end{array}$ & $7(\mathrm{~A})$ & 2,33 & 24 \\
\hline
\end{tabular}




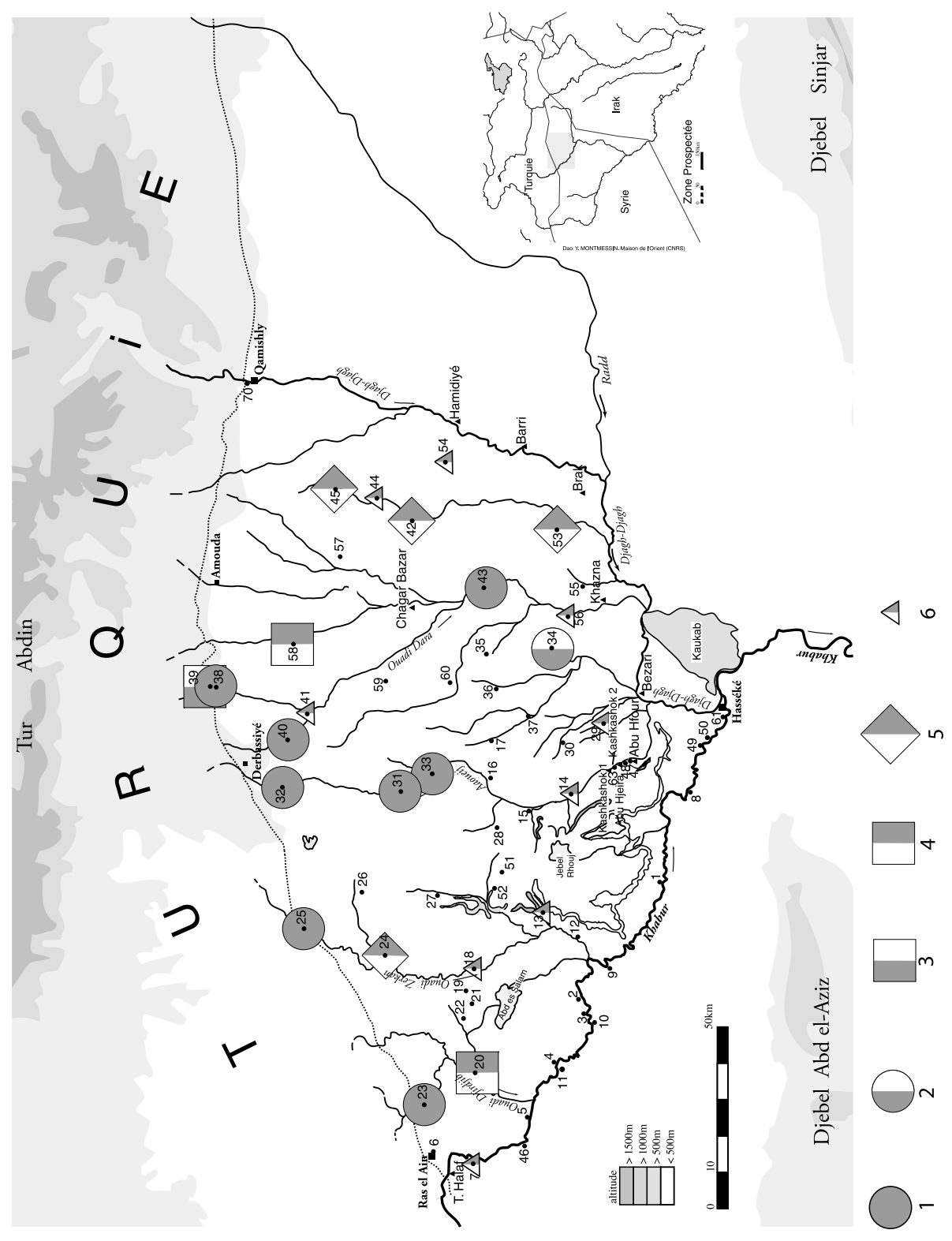

Fig. 1 | Distribution of settlements during the Old Babylonian period according to material from the survey.

I- The ro most densely occupied sites, yielding the largest amount of sherds in the two studies (sherds retained and sherds collected).

2- The Io most densely occupied sites, yielding the largest amount of sherds in the study of sherds retained.

3- The Io most densely occupied sites from the study of sherds retained.

4- The io most densely occupied sites from the study of sherds collected.

5- The ro sites yielding most sherds from sherds collected.

6- Other sites occupied according to the study of sherds collected. 


\section{B.2 The Mittani Period (Table V and Fig. 2)}

In this period the number of settled sites -53 in total - is more than double than that of the preceding period. Furthermore, we find that the sites are distributed across the entire region included in our survey. But the total number of collected potsherds (4555) is almost the same as for the previous period (45I7), which suggests that these sites were on the whole comparatively small in population. Another major difference is that no settlement noticeably exceeds the others in quantity of potsherds collected. Two sites yielded between 400 and 350 potsherds for this period (T. 42 and 38), seven sites between 3 I5 and 226 sherds (T. $45,33,28,24,32,25,34)$, I5 more between I50 and 60 potsherds (T. 2, 40, 7, 54, 70, 37, 4, 44, I8, 30, 4I, 43, 5I, I3, 53) and the last 29 each yielded less than 50 potsherds each.

T. 3I, which seems to have had a large population in the preceding period, disappears entirely. Faivre's identification of certain potsherds characteristic of the late Khabur/early Mittani, indicates that this site was probably abandoned around the beginning of the Mittani period (see Table II above). The other six sites, which in the preceding period had yielded large quantities of potsherds, once again come out on top of the ranking (in descending order, they are T. 42, 38, 45, 33, 32, 25), but we also see the arrival of two new sites in this group, T. 28 and 24 .

Most remarkably, several new sites appear along the Khabur, although only T. 4 makes an honorable showing (sixth) in the population density rankings, and only T. 2 does well (tenth) in terms of potsherds collected. All the other settlements along the river seem to have small populations, a fact which does not seem compatible with the supposed presence nearby of one of the regional capitals.

On the whole, this period is characterized by a proliferation of small settlements or sites with a relatively low population density covering the entirety of the surveyed area. The four most densely populated sites are smaller than 0.5 ha. The sites with the largest geographic area (ca. 4 or 5 ha) only come in at eighteenth or nineteenth for density (T. 33 and 45). This indicates that a major change must have occurred from the preceding period, even though the largest sites are still mostly located in the northern zone. 
Table V | List of sites, which yielded potsherds dating to the Mittani period, based on the analysis of all potsherds originally collected. Sites are ordered by quantity of potsherds collected (left column) and by order of population density measured as a ratio of quantity to surface (right column).

\begin{tabular}{|c|c|c|c|c|c|c|}
\hline \multicolumn{2}{|c|}{$\begin{array}{l}\text { Ranking of sites according } \\
\text { to number of collected pot- } \\
\text { sherds }\end{array}$} & \multirow[t]{2}{*}{ Drainage Basin } & \multirow[t]{2}{*}{ Surface } & \multirow{2}{*}{$\begin{array}{l}\text { Number of } \\
\text { collected } \\
\text { potsherds of } \\
\text { the Khabur } \\
\text { Period } \\
\text { Total = } 4555\end{array}$} & \multicolumn{2}{|c|}{$\begin{array}{l}\text { Ranking according to } \\
\text { population density }\end{array}$} \\
\hline Ranking & Site & & & & $\begin{array}{l}\text { Number of } \\
\text { pot- } \\
\text { sherds/ha }\end{array}$ & Ranking \\
\hline I & 42 (Tell Arbid) & $\begin{array}{l}\text { Unnamed Wadi / } \\
\text { Jaghjagh }\end{array}$ & 7 ha & 402 & 57,42 & 22 \\
\hline 2 & $\begin{array}{l}38 \text { (Tell Aïn el } \\
\text { Qurd) }\end{array}$ & Wadi Dara & 2,42 ha & 353 & 145,86 & 8 \\
\hline 3 & 45 (Tell Ahmar) & $\begin{array}{l}\text { Unnamed Wadi / } \\
\text { Jaghjagh }\end{array}$ & $4,9 \circ$ ha & $3 \mathrm{I} 5$ & 64,28 & I9 \\
\hline 4 & 33 (Tell Hanoua) & Aoueij, left bank & ca. 3,90 ha & 285 & 73,07 & I8 \\
\hline 5 & 28 (Tell Hassek) & Aoueij, right bank & I, $5 \circ$ ha & 270 & I80 & 7 \\
\hline 6 & 24 (Tell Hermel) & Wadi Zerkan & 2 ha & 250 & I25 & 12 \\
\hline 7 & 32 (Tell Kdich) & Aoueij, right bank & o,8० ha & 230 & 287,5 & $\mathbf{I}$ \\
\hline 8 & 25 (Tell Arade) & Wadi Zerkan & I ha & 228 & 228 & 5 \\
\hline 9 & 34 (Tell Atach) & $\begin{array}{l}\text { Unnamed Wadi / } \\
\text { Jaghjagh }\end{array}$ & 2 ha & 226 & II3 & I4 \\
\hline IO & $\begin{array}{l}2 \text { (Tell Umm } \\
\text { Urhafa) }\end{array}$ & Khabur, left bank & I, $5 \circ$ ha & I47 & 98 & 16 \\
\hline II & 40 (Tell Qattine) & $\begin{array}{l}\text { Unnamed Wadi / } \\
\text { Dara }\end{array}$ & 0,50 ha & II9 & 238 & 3 \\
\hline 12 & 7 (Tell Qattine) & Khabur, right bank & 5 ha & 98 & I9,6 & 37 \\
\hline I3 & $\begin{array}{l}54 \text { (Guir Zil } \\
\text { Kabir) }\end{array}$ & $\begin{array}{l}\text { Unnamed Wadi / } \\
\text { Jaghjagh }\end{array}$ & 7 ha & 96 & I6 & 40 \\
\hline 14 & 70 (Tell Badan) & $\begin{array}{l}\text { Jaghjagh, right } \\
\text { bank }\end{array}$ & I7,50 ha & 96 & 5,48 & 47 \\
\hline
\end{tabular}




\begin{tabular}{|c|c|c|c|c|c|c|}
\hline \multicolumn{2}{|c|}{$\begin{array}{l}\text { Ranking of sites according } \\
\text { to number of collected pot- } \\
\text { sherds }\end{array}$} & \multirow[t]{2}{*}{ Drainage Basin } & \multirow[t]{2}{*}{ Surface } & \multirow{2}{*}{$\begin{array}{l}\begin{array}{l}\text { Number of } \\
\text { collected } \\
\text { potsherds of } \\
\text { the Khabur } \\
\text { Period }\end{array} \\
\text { Total }=4555\end{array}$} & \multicolumn{2}{|c|}{$\begin{array}{l}\text { Ranking according to } \\
\text { population density }\end{array}$} \\
\hline Ranking & Site & & & & $\begin{array}{l}\text { Number of } \\
\text { pot- } \\
\text { sherds/ha }\end{array}$ & Ranking \\
\hline 15 & 37 (Tell Bati) & $\begin{array}{l}\text { Wadi Rijla / } \\
\text { Jaghjagh }\end{array}$ & $\begin{array}{l}5 \text { ha, but } \\
\text { settled } \\
\text { area only } \\
=\mathrm{I}, 82 \text { ha } 37\end{array}$ & $\begin{array}{l}96 \text { (mainly } \\
\text { A, B, G, H, a } \\
\text { bit in E, F, } \\
\text { none in C, } \\
\text { D) }\end{array}$ & 52,74 & 24 \\
\hline 16 & $\begin{array}{l}4 \text { (Tell Umm el } \\
\text { Asafir) }\end{array}$ & Khabur, left bank & 0,50 ha & 96 & 192 & 6 \\
\hline I7 & $\begin{array}{l}44 \text { (Guir } \\
\text { Diouane) }\end{array}$ & $\begin{array}{l}\text { Unnamed Wadi / } \\
\text { Jaghjagh }\end{array}$ & ०,8० ha & 88 & IIO & 15 \\
\hline I8 & $\begin{array}{l}\text { I8 (Tell el Ward } \\
\text { Charqi) }\end{array}$ & Wadi Zerkan & ca. I ha & 80 & 80 & I7 \\
\hline 19 & $\begin{array}{l}30 \text { (Tell Berguil } \\
\text { Buz) }\end{array}$ & $\begin{array}{l}\text { Unnamed Wadi / } \\
\text { Jaghjagh }\end{array}$ & 0,60 ha & 79 & I3I, 66 & IO \\
\hline 20 & 4I (Tell Baqar) & $\begin{array}{l}\text { Unnamed Wadi / } \\
\text { Dara }\end{array}$ & 2,20 ha & 70 & 3I,8I & 28 \\
\hline $2 I$ & 43 (Tell Farho) & Wadi Dara & ca. $0,3 \circ$ ha & 70 & 233,33 & 4 \\
\hline 22 & $\begin{array}{l}\text { 5I (Tell Rhazal } \\
\text { Tahtani) }\end{array}$ & $\begin{array}{l}\text { Unnamed Wadi / } \\
\text { Zerkan }\end{array}$ & I, 50 ha & 65 & 43,33 & 25 \\
\hline 23 & $\begin{array}{l}\text { I3 (Tell Aïn el } \\
\text { Abed) }\end{array}$ & $\begin{array}{l}\text { Wadi Zerkan / } \\
\text { source }\end{array}$ & 0,50 ha & 65 & I30 & II \\
\hline 24 & 53 (Tell Kourdiss) & $\begin{array}{l}\text { Unnamed Wadi / } \\
\text { Jaghjagh }\end{array}$ & 2 ha & 6I & 30,5 & 29 \\
\hline 25 & I4 (Tell Jamilo) & Aoueij, left bank & 8 ha & 43 & 5,37 & $4^{8}$ \\
\hline 26 & 56 (Tell Nurek) & Wadi Dara & 6 ha & 42 & 7 & 44 \\
\hline 27 & I (Tell Rommane) & Khabur, left bank & $2,5 \circ$ ha & 42 & $\mathrm{I} 6,8$ & 39 \\
\hline 28 & $\begin{array}{l}35 \text { (Tell Avguir } \\
\text { Fawqani) }\end{array}$ & $\begin{array}{l}\text { Unnamed Wadi/ } \\
\text { Dara }\end{array}$ & 0,30 ha & 35 & II6,66 & 13 \\
\hline
\end{tabular}

37 Note that the central part of T. 37, on which houses and a road had been built, was not surveyed, hence the difference between the total size of the site and the size of the settled area in this period. 


\begin{tabular}{|c|c|c|c|c|c|c|}
\hline \multicolumn{2}{|c|}{$\begin{array}{l}\text { Ranking of sites according } \\
\text { to number of collected pot- } \\
\text { sherds }\end{array}$} & \multirow[t]{2}{*}{ Drainage Basin } & \multirow[t]{2}{*}{ Surface } & \multirow{2}{*}{$\begin{array}{l}\text { Number of } \\
\text { collected } \\
\text { potsherds of } \\
\text { the Khabur } \\
\text { Period } \\
\text { Total = } 4555\end{array}$} & \multicolumn{2}{|c|}{$\begin{array}{l}\text { Ranking according to } \\
\text { population density }\end{array}$} \\
\hline Ranking & Site & & & & $\begin{array}{l}\text { Number of } \\
\text { pot- } \\
\text { sherds/ha }\end{array}$ & Ranking \\
\hline 29 & $\begin{array}{l}60 \text { (Tell } \\
\text { Tcholama } \\
\text { Tahtani) }\end{array}$ & $\begin{array}{l}\text { Unnamed Wadi/ } \\
\text { Dara }\end{array}$ & 6 ha & 35 & 5,83 & 45 \\
\hline 30 & $\begin{array}{l}57 \text { (Tell Chil } \\
\text { Virane) }\end{array}$ & $\begin{array}{l}\text { Without wadi / } \\
\text { Dara }\end{array}$ & I,I5 ha & 34 & 29,56 & 30 \\
\hline $3 \mathrm{I}$ & I7 (Tell Effendi) & $\begin{array}{l}\text { Unnamed Wadi / } \\
\text { Jaghjagh }\end{array}$ & $\begin{array}{l}\text { I,9० ha, } \\
\text { but the } \\
\text { settled } \\
\text { area only } \\
=0,84 \text { ha }\end{array}$ & $\begin{array}{l}32(\mathrm{~A}, \mathrm{~B}, \mathrm{C} \\
\mathrm{F})\end{array}$ & 38,09 & 26 \\
\hline 32 & $\begin{array}{l}\text { 2I (Tell Baarair } \\
\text { Kabir) }\end{array}$ & $\begin{array}{l}\text { Without wadi / } \\
\text { Jirjib }\end{array}$ & 0,85 ha & 30 & 35,29 & 27 \\
\hline 33 & $\begin{array}{l}22 \text { (Tell Ed } \\
\text { Daudiye) }\end{array}$ & $\begin{array}{l}\text { Without wadi / } \\
\text { Jirjib }\end{array}$ & I, 50 ha & 30 & 20 & 36 \\
\hline 34 & II (Tell Atchach) & Khabur, right bank & I ha & 29 & 29 & $3 I$ \\
\hline 35 & 58 (Tell Dukri) & $\begin{array}{l}\text { Unnamed Wadi / } \\
\text { Dara }\end{array}$ & $\begin{array}{l}0,30 \text { ha, } \\
\text { but A only } \\
=0,20 \text { ha }\end{array}$ & $28(\mathrm{~A})$ & $\mathrm{I} 4 \mathrm{O}$ & 9 \\
\hline 36 & $\begin{array}{l}5 \text { (Tell Asnane } \\
\text { Fawqani) }\end{array}$ & Khabur, left bank & I, 50 ha & 27 & I8 & 38 \\
\hline 37 & 50 (Tell Mujarja) & Khabur, left bank & 0,50 ha & 27 & 54 & 23 \\
\hline $38-$ & I5 (Tell Beydar) & Aoueij, right bank & $\begin{array}{l}\mathrm{I} 4,50 \text { ha } \\
\text { but the } \\
\text { settled } \\
\text { area only } \\
=\mathrm{I} 2,90 \mathrm{ha}\end{array}$ & $\begin{array}{l}26 \\
\text { (everywhere } \\
\text { except C, D) }\end{array}$ & $2, \mathrm{OI}$ & 53 \\
\hline $39-$ & $\begin{array}{l}23 \text { (Tell Alouq } \\
\text { Charqi) }\end{array}$ & Jirjib Gharbi & o,ro ha & 25 & 250 & 2 \\
\hline $40-$ & 20 (Tell Jamus) & Jirjib & 0,40 ha & 25 & 62,5 & 20 \\
\hline $4 \mathrm{I}^{-}$ & $\begin{array}{l}27 \text { (Tell ech } \\
\text { Chour Gharbi) }\end{array}$ & $\begin{array}{l}\text { Unnamed Wadi / } \\
\text { Zerkan }\end{array}$ & 6 ha & 24 & 4 & 49 \\
\hline
\end{tabular}




\begin{tabular}{|c|c|c|c|c|c|c|}
\hline \multicolumn{2}{|c|}{$\begin{array}{l}\text { Ranking of sites according } \\
\text { to number of collected pot- } \\
\text { sherds }\end{array}$} & \multirow[t]{2}{*}{ Drainage Basin } & \multirow[t]{2}{*}{ Surface } & \multirow{2}{*}{$\begin{array}{l}\text { Number of } \\
\text { collected } \\
\text { potsherds of } \\
\text { the Khabur } \\
\text { Period } \\
\text { Total = } 4555\end{array}$} & \multicolumn{2}{|c|}{$\begin{array}{l}\text { Ranking according to } \\
\text { population density }\end{array}$} \\
\hline Ranking & Site & & & & $\begin{array}{l}\text { Number of } \\
\text { pot- } \\
\text { sherds/ha }\end{array}$ & Ranking \\
\hline $42-$ & $\begin{array}{l}29 \text { (Tell Aswad } \\
\text { Tahtani) }\end{array}$ & $\begin{array}{l}\text { Unnamed Wadi / } \\
\text { Jaghjagh }\end{array}$ & $\begin{array}{l}\text { I ha, but } \\
\text { the settled } \\
\text { area only } \\
=0,33 \text { ha }\end{array}$ & $\begin{array}{l}20(\mathrm{~A} \\
\text { mainly, and } \\
\mathrm{B}, \mathrm{C})\end{array}$ & 60,60 & $2 \mathrm{I}$ \\
\hline $43^{-}$ & 6 (Tell Fakhariya) & Khabur, left bank & 3,50 ha & 20 & $5,7 \mathrm{I}$ & 46 \\
\hline $44^{-}$ & $\begin{array}{l}\text { I2 (Tell Ashnane } \\
\text { Charqi) }\end{array}$ & Wadi Zerkan & $0,75 \mathrm{ha}$ & I6 & $2 \mathrm{I}, 33$ & 35 \\
\hline $45^{-}$ & I6 (Tell Khatun) & Aoueij, left bank & $\mathrm{I}, 20 \mathrm{ha}$ & I5 & $\mathrm{I} 2,5$ & 42 \\
\hline 46- & 3 (Tell Tawil) & Khabur, left bank & I ha & $\mathrm{I} 4$ & $\mathrm{I} 4$ & $4 \mathrm{I}$ \\
\hline $47^{-}$ & 48 (unnamed) & Aoueij, right bank & 0,50 ha & II & 22 & 34 \\
\hline $48-$ & 26 (Tell Dabach) & $\begin{array}{l}\text { Wadi Seher / } \\
\text { Zerkan }\end{array}$ & $\begin{array}{l}3,50 \text { ha } \\
\text { but the } \\
\text { settled } \\
\text { area only } \\
=\text { ca. I ha }\end{array}$ & $\begin{array}{l}\text { Io (D, F, G, } \\
\text { H) }\end{array}$ & IO & 43 \\
\hline $49-$ & $\begin{array}{l}47 \text { (actual Tell } \\
\text { Abu Hufur) }\end{array}$ & Aoueij, right bank & 2,60 ha & 9 & 3,46 & $5 I$ \\
\hline 50- & 8 (Tell Majdel) & Khabur, right bank & $\begin{array}{l}25 \text { ha but } \\
\text { the settled } \\
\text { area only } \\
=3,70 \text { ha }\end{array}$ & $9(\mathrm{~A}, \mathrm{~B})$ & 2,43 & 52 \\
\hline $5 \mathrm{I}^{-}$ & Io (Tell Tawila) & Khabur, right bank & I, 60 ha & 6 & 3,75 & 50 \\
\hline $52-$ & $\begin{array}{l}9 \text { (Tell Chamrane } \\
\text { Tahtani) }\end{array}$ & Khabur, right bank & 0,16 ha & 4 & 25 & 33 \\
\hline 53- & II (unnamed) & Khabur, right bank & 0,07 ha & 2 & 28,57 & 32 \\
\hline
\end{tabular}




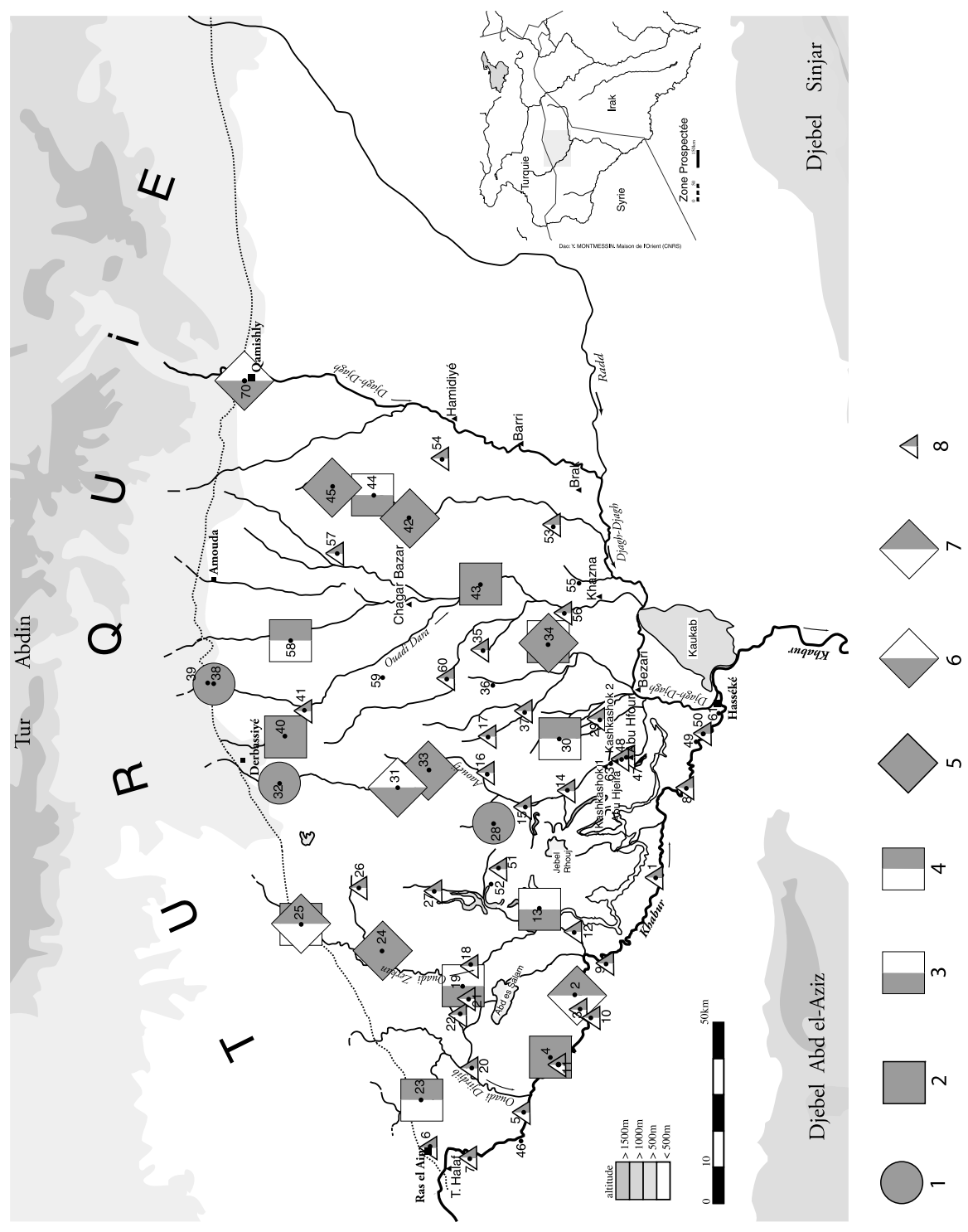

Fig. 2 | Distribution of settlements during the Mittani period according to material from the survey.

I- The Io most densely occupied sites, yielding the largest amount of sherds in the two studies (sherds retained and sherds collected).

2- The io most densely occupied sites from the two studies.

3- The Io most densely occupied sites from the study of sherds retained.

4- The ro most densely occupied sites from the study of sherds collected.

5- The Io sites yielding most sherds from the two studies.

6 - The Io sites yielding the largest amount of sherds from sherds retained

7- The Io sites yielding the largest amount of sherds from sherds collected

8- Other sites occupied according to the study of sherds collected. 


\section{Conclusion}

The data of our field survey have been presented here from different points of view (potsherds retained, potsherds collected, total quantity of potsherds by site and density by hectare). In the end, the results obtained by each of these methods differ little from each other and show a distinct change in the demographics of the region between the "Khabur period" and the Mittani period. The number of sites evolves considerably from one period to the other, and we see clearly new settlements on the banks of the Khabur from the middle of the second millennium onward. However, the population during the Mittani period is clearly dispersed in small villages, as none of the sites surveyed are of urban character (determined by the size and quantity of potsherds). In addition, there seems to be no way of grouping of these villages around a possible undiscovered major site; this contradicts the conventional hypothesis that villages usually crop up as satellites of a major city, and once again raises the unanswered question of the real locations of Waššukanni and Taidu.

Scholars have often discussed the forced resettlement in this region during the Assyrian periods, in particular in the Neo-Assyrian period, but up until now our knowledge of the earlier demographics of the Khabur area was very limited. In isolation, the data of this field survey are not enough to determine definitively the causes for resettlement during the Mittani period: these may include the voluntary or forced settlement of previously nomadic groups, the arrival of new populations, etc. These are all possibilities that the textual documentation will have to clarify. Nevertheless, the fact that the total quantity of potsherds collected is approximately the same for the two periods suggests that there was no general increase in the population between the two periods, but rather a significant change in its distribution. The improvement of climatic conditions in this period probably played a decisive role in the dispersal of the population into small villages over the whole area of the survey.

\section{Bibliography}

Bretschneider 1997

J. Bretschneider, Die Unterstadt (Feld J), in: M. Lebeau - A. Suleiman (eds.), Tell Beydar, Three Seasons of Excavations (I992-I994). A Preliminary Report, Subartu 3 (Turnhout I997) 209-230.

\section{Courty 1994}

M.-A. Courty, Le cadre paléogéographique des occupations humaines dans le bassin du Haut-Khabur (Syrie du Nord-Est), Premiers résultats, Paléorient 20/I, I994, 2I-59.

\section{Dillemann 1962}

L. Dillemann, Haute-Mésopotamie orientale et pays adjacents. Contribution à la géographie historique de la région, du $\mathrm{V}^{\mathrm{e}} \mathrm{s}$. avant l'ère chrétienne au $\mathrm{VI}^{\mathrm{e}} \mathrm{s}$. de cette ère, Bibliothèque Archéologique et Historique 72 (Paris I962). 


\section{Durand 1998}

J.-M. Durand, Les Bédouins, ch. IX in: Documents épistolaires du palais de Mari, t. II, Littératures Anciennes du Proche-Orient (Paris I998) 4I7-5II.

\section{Durand 2004}

J.-M. Durand, Peuplement et sociétés à l'époque amorrite: (I) les clans bensim'alites, in: C. Nicolle (ed.), Nomades et sédentaires dans le Proche-Orient ancien, Amurru 3 (Paris 2004) III-I97.

\section{Eidem - Warburton 1996}

J. Eidem - D. Warburton, In the Land of Nagar. A Survey around Tell Brak, Iraq 58, 1996, $5 \mathrm{I}-64$.

\section{Faivre 1999}

X. Faivre, Histoire du peuplement du "Triangle du Habur" au Bronze Moyen et au début du Bronze Récent: étude céramologique d'après les fouilles de Tell Mohammed Diyab (1987-1996) et la prospection dans le bassin occidental du Haut-Habur (I989-I997), 4 vols. (Thèse de Doctorat, Université de Lille III I999).

\section{Fielden I98I}

K. Fielden, The Chronology of Settlement in Northeastern Syria during the Later Fourth and Third Millennia B.C. in the Light of Ceramic Evidence from Tell Brak (British Doctoral Thesis, Faculty of Oriental Studies, Corpus Christi College, Oxford, 2 vols., I98I).

\section{Haas - Wäfler 1985}

V. Haas - M. Wäfler, Möglichkeiten der Identifizierung des Tall al-Hamidiya, in: Tall al-Hamidiya I, Vorbericht I984, OBO Series Archaeologica 4, I985, 53-73.

Hole 2002-2003

F. Hole, Khabur Basin Project, I986-200I, Annales Archéologiques Arabes Syriennes 45-46, 2002-2003, II-20.

\section{Koliński 2007}

R. Koliński, Sir Max Mallowan's Excavations at Tell Arbid in I936, Iraq 69, 2007, 73-II5.

\section{Kulemann-Ossen 2009}

S. Kulemann-Ossen, Zum Siedlungsgeschehen des 2. Jahrtausends v. Chr. am Unteren Habur, in: E. Cancik-Kirschbaum - N. Ziegler (eds.), Entre les Fleuves, I, Untersuchungen zur historischen Geographie Obermesopotamiens im 2. Jahrtausend v. Chr., Berliner Beiträge zum Vorderen Orient 20, 2009, I5I-I63.

\section{van Liere - Lauffray I954-I955}

W. J. van Liere - J. Lauffray, Nouvelle prospection archéologique dans la Haute Jézireh syrienne, Annales Archéologiques de Syrie 4/5, I954-55, I29-148.

\section{Lyonnet 1992}

B. Lyonnet, Reconnaissance dans le Haut-Khabur: étude de la céramique, in: J.-M. Durand (ed.), Mémoires de N.A.B.U. 2, Recherches en Haute-Mésopotamie. Tell Mohammed Diyab campagnes I990 et I99I, SEPOA (Paris I992) I03-I32. 


\section{Lyonnet 1996}

B. Lyonnet, La prospection archéologique de la partie occidentale du Haut-Habur (Syrie du N.E.): méthodes, résultats et questions autour de l'occupation aux $3^{\mathrm{e}}$ et $2^{\mathrm{e}}$ millénaires av. n. è., in: J.-M. Durand (ed.), Mari, Ebla et les Hourrites, Amurru I, ERC (Paris I996) 363-376.

\section{Lyonnet 1998}

B. Lyonnet, Le peuplement de la Djéziré occidentale au début du $3^{\mathrm{e}}$ millénaire, villes circulaires et pastoralisme: questions et hypothèses, Subartu 4/I (Turnhout I998) I79-I93.

\section{Lyonnet 2000 a}

B. Lyonnet, Pemière partie. Présentation générale de la prospection, in: B. Lyonnet (ed.), Prospection archéologique du Haut-Khabur occidental (Syrie du N.E.), volume I. Bibliothèque Archéologique et Historique, T. I55 (Beyrouth 2000) 5-73.

\section{Lyonnet 200ob}

B. Lyonnet, Prospection archéologique du Haut-Khabur occidental (Syrie du N.E.), volume I. Bibliothèque Archéologique et Historique, T. I55 (Beyrouth 2000).

\section{Lyonnet 200I}

B. Lyonnet, L'occupation des marges arides de la Djéziré: pastoralisme et nomadisme aux débuts du $3^{\mathrm{e}}$ et du $2^{\mathrm{e}}$ millénaire - II, in: B. Geyer (ed.), Conquête de la steppe et appropriation des terres sur les marges arides du Croissant fertile, Travaux de la Maison de l'Orient et de la Méditerranée 36 (Lyon 200I) 15-26.

\section{Lyonnet 2004}

B. Lyonnet, Le nomadisme et l'archéologie: problèmes d'identification. Le cas de la partie occidentale de la Djéziré aux zème et début du zème mill. av. n. è., in: C. Nicolle (ed.), Nomades et sédentaires dans le Proche-Orient ancient, Amurru 3 (Paris 2004) 25-49.

\section{Lyonnet 2009}

B. Lyonnet, Who Lived in the 3rd Millennium "Round Cities" of Northern Syria?, in: J. Szuchman (ed.), Nomads, Tribes, and the State in the Ancient Near East: Cross-Disciplinary Perspectives, Oriental Institute Seminars 5 (Chicago 2009) I79-200.

\section{Mallowan 1936}

M. E. L. Mallowan, The Excavations at Chagar Bazar and an Archaeological Survey of the Habur Region, I934-I935, Iraq 3, I936, I-86.

\section{Mallowan 1937}

M. E. L. Mallowan, The Excavations at Chagar Bazar and an Archaeological Survey of the Habur Region, second campaign i936, Iraq 4, I937, 9I-I77.

\section{Mc Ewan et al. 1958}

C. Mc Ewan - L. Braidwood - H. Frankfort - H. G. Güterbock - R. C. Haines - H. J. Kantor C. H. Kraeling, Soundings at T. Fakhariyah, The University of Chicago Oriental Institute Publications vol. 79 (Chicago I958).

\section{Meijer I986}

D. Meijer, A Survey in Northeastern Syria, Publication of the Nederlands Historich-Archaeologisch Instituut Te Istanbul 58 (Istanbul I986). 


\section{Monchambert 1984}

Y. Monchambert, Le futur lac du Moyen-Khabour: rapport sur la prospection archéologique menée en I983, Syria 6I, I984, I8I-2I8.

\section{Nieuwenhuyse 2000}

O. Nieuwenhuyse, Halaf Settlement in the Khabur Headwaters, in: B. Lyonnet (ed.), Prospection archéologique du Haut-Khabur occidental (Syrie du N.E.), volume I. Bibliothèque Archéologique et Historique I55 (Beyrouth 2000) I5I-260.

\section{Nieuwenhuyse - Wilkinson 2008}

O. Nieuwenhuyse - T. Wilkinson, Late Neolithic Settlement in the Area of Tell Beydar (NE Syria), in: M. Lebeau - A. Suleiman (eds.), Beydar Studies I, Subartu 2I (Turnhout 2008) 268-303.

\section{Oates 1977}

D. Oates, The Excavations at T. Brak, I976, Iraq 39, I977, 233-244 et pl. IV-X.

\section{Oates 1987}

J. Oates, A Note on 'Ubaid' and Mitanni Pottery from Tell Brak, Iraq 49, I987, I93-198.

\section{Opitz 1927}

D. Opitz, Die Lage von Wassuganni, Zeitschrift für Assyriologie und verwandte Gebiete vol. 37, I927, 299-301.

\section{Oppenheim I931}

M. A. S. von Oppenheim, Der Tell Halaf: Eine neue Kultur im ältesten Mesopotamien (Berlin I93I).

\section{Poidebard 1934}

A. Poidebard (Père), La Trace de Rome dans le désert de Syrie. Le limes de Trajan à la conquête arabe. Recherches aériennes (1925-1932), Bibliothèque Archéologique et Historique 18,2 vols. (Paris I934).

\section{Ristvet - Weiss 2005}

L. Ristvet - H. Weiss, The Habur Region in the Late Third and Early Second Millennium B.C., in: W. Orthmann (ed.), The History and Archaeology of Syria vol. I (Saarbrücken 2005).

\section{Röllig - Kühne $1977-78$}

W. Röllig - H. Kühne, The Lower Khabur. A Preliminary Report on a Survey conducted by the Tübinger Atlas des Vorderen Orients in 1975, Annales Archéologiques Arabes Syriennes 27-28, I977-78, II5-I40.

\section{Röllig - Kühne 1983}

W. Röllig - H. Kühne, The Lower Khabur. $2^{\text {nd }}$ Preliminary Report on a Survey in I977, Annales Archéologiques Arabes Syriennes 32, tome 2, I983, I87-199.

\section{Shibata 2009}

D. Shibata, An Old Babylonian Manuscript of the Weidner God list from Tell Taban, Iraq 7I, 2009, 33-42. 


\section{Stein - Wattenmaker 1990}

G. Stein - P. Wattenmaker, The Tell Leilan Regional Survey: Preliminary Report, in: N. Miller (ed.), Economy and Settlement in the Near East. Analyses of Ancient Sites and Materials, MASCA Papers in Science and Archaeology 7 (supplement) (Philadelphia I990) 9I8.

\section{Ur - Wilkinson 2008}

J. Ur - T. Wilkinson, Settlement and Economic Landscapes of Tell Beydar and its Hinterland, in: M. Lebeau - A. Suleiman (eds.), Beydar Studies I, Subartu 2I (Turnhout 2008) 305-327.

\section{Wilkinson 2000}

T. Wilkinson, Archaeological Survey of the Tell Beydar Region, Syria, I997. A Preliminary Report, Subartu 6 (Turnhout 2000) I-37. 
